\title{
Optimal Liquidity management and Hedging in the presence of a Non-Predictable Investment Opportunity
}

\author{
Stéphane Villeneuve*Xavier Warin ${ }^{\dagger}$
}

October 22, 2013

\begin{abstract}
In this paper, we develop a dynamic model that captures the interaction between a firm's cash reserves, the risk management policy and the profitability of a nonpredictable irreversible investment opportunity. We consider a firm that has assets in place generating a stochastic cash-flow stream. The firm has a non-predictable growth opportunity to expand its operation size by paying a sunk cost. When the opportunity is available, the firm can finance it either by cash or by costly equity issuance. We provide an explicit characterization of the firm strategy in terms of investment, hedging, equity issuance and dividend distribution.
\end{abstract}

\section{Introduction}

Both corporate liquidity management and hedging policy have been the topic of a large academic literature in the last thirty years. The literature aimed to depart from the benchmark model of perfect capital markets (Modigliani and Miller, [14]) to explain why in practice the management of cash holdings and hedging are key determinants of a firm's success. Several directions have been explored for explaining how and why firms should hold cash reserves and hedge their risks. The literature has mainly focused on the precautionary demand of cash holdings in order to both meet the operational needs and avoid a costly outside fund raising in financial distress. Empirical studies have confirmed the precautionary role of cash holdings by documenting that cash holdings represent a significant and growing share of corporate wealth (Berk and DeMarzo [3] and Bates, Kahle, and Stulz [2]).

On the other hand, the literature on corporate finance has somewhat neglected the importance of cash holdings and hedging in the determination of the optimal decision to undertake an irreversible investment. More precisely, while it is clear from the pecking order theory that firms prefer to use cash holdings to finance investment in order to avoid the costs of external financing, few papers have concentrated on the order of magnitude of the self-financing investment that firm should optimally allocate in a dynamic setting. Up to now, the real option theory of irreversible investment under uncertainty has assumed

${ }^{*}$ Toulouse School of Economics (CRM, IDEI) Manufacture des tabacs 22 allée de brienne 31000 Toulouse and Market Risk and Creation Value an initiative from SCOR under the aegis of Fondation du risque.

${ }^{\dagger}$ EDF R\&D \& FiME, Laboratoire de Finance des Marchés de l'Energie (www.fime-lab.org) 
that outside funds can be raised at no cost to finance investment opportunity. As a consequence, the decision to invest is made independently of the firm cash holdings and of the firm capital structure (see Dixit and Pindyck [8] for a survey). There are few papers that model the role of cash reserves in the optimal decision to expand. The first attempt has been made by Boyle and Guthrie [5] that considers a liquidity constrained firm that must finance the investment internally. As a continuation of Boyle and Guthrie's work, Decamps and Villeneuve [7] study the interaction between dividend policy and investment decision in a growth opportunity of a liquidity constrained firm that has no access to external funds. They characterize situations where it is optimal to postpone dividend distribution in order to invest at a subsequent date in the growth opportunity. Asvanunt, Broadie and Sundaresan (2010) develop a structural model that captures the interaction between the cash reserves and investment opportunity for a firm that has some debt outstanding. Finally, Guo and Pham [10] consider a model where a company has an option to invest in a way of producing a good and can continuously adjust its production capacity.

Most importantly, our study is very related to the paper by Hugonnier, Malamud and Morellec [12] that considers the interactions between cash holdings, dividend distribution and capacity expansion when firms face uncertainty regarding their ability to raise external funds and have to pay a search cost to meet outside investors. The novel part of our paper is both incorporating the possibility of hedging and assuming that our firm's production function exhibits constant return to scale and thus the growth opportunity affects both the profitability and the risk as in Bolton, Chen and Wang [4]. Unlike [7] and [12], we also assume that the firm has access to costly external financing whenever it is needed and that the decision maker does not have a monopoly on the investment opportunity. We are working in the opposite situation where we assume perfect competition between firms and thus the growth opportunity is a take-it-or-leave-it offer.

In this paper, we develop a simple model in continuous time whose objective is to capture the dual role of cash holdings and hedging decisions. The first one is the welldocumented precautionary role of cash reserves which provide liquidity in financial distress while the second is the frictionless financing of investment opportunities. Our analysis shows that when capital supply is costly, irreversible real investment decision and financing depend on the firm's cash holdings. Poor-cash firms may be reluctant to invest in a growth opportunity because they anticipate future financing constraints regardless of the source of financing. In our model, the firm's manager has to make three interrelated decisions: how much cash to hold, whether to hedge and whether to invest with internal or external funds. The firms find it optimal to hold cash for two motives. First, cash holdings can be used to cover operating losses. Second, cash holdings can be used to invest in a growth opportunity. We prove that even in the case where the firm maximizes its access to external financing by choosing optimally the level of its internal funds, it could happen that the firm refuses to undertake an option to expand because investment increases the liquidity risk.

Finally, the prediction of our model in terms of hedging depends on the intensity of the opportunity arrival. Traditionally, because cash holdings are used to avoid inefficient closure, the shareholder value function is concave which implies a willingness to decrease the level 
of uncertainty by using hedging instruments (see Højgaard and Taksar [11] for a rigorous treatment of this result). For a large set of parameters, we find that the concavity feature still holds in our model in the presence of a growth opportunity. As a consequence, the firm is willing to buy hedging and the level of hedging policy is a decreasing function of the level of cash holdings. Poor-cash firms are more ready to hedge and the firm's manager ceases to buy hedging instruments when the cash holdings are close to the threshold above which dividend distribution is optimal. The novel implication of our model is given by situation where the continuation shareholder value after the arrival of the investment opportunity exhibits local convexity. The fact that the firm value may exhibit local convexity has been already stated and proved in Decamps and Villeneuve [7] and Hugonnier, Malamud and Morellec [12] but the contribution of our paper is twofold. First, we propose a rather general methodology based on the maximum principle to characterize the value function and the optimal policy, and we focus on the hedging decision resulting from the local convexity property. In the high-intensity case, it is interesting to note that the firm hedging policy before the arrival of the opportunity is non monotonic. The shareholders gamble by ceasing to hedge in order to increase cash holdings to make the growth option valuable.

Using the dynamic programming principle, we solve the mixed regular/singular control problem faced by the firm's manager into a two-stage procedure. After the arrival date of the investment opportunity, we solve explicitly the two control problems associated respectively to the decision to invest or not. We have to solve explicitly (Proposition 3.4) a non linear Hamilton-Jacobi-Bellman variational inequalities and to validate the optimality of this solution by a verification theorem (Proposition 3.3). Before the arrival date of the investment opportunity, we have to solve a non standard mixed regular/singular control problem whose value is known at a stopping time $T$ corresponding to the arrival of the investment opportunity. The paper is organized as follows. Section 2 presents the model and describes the decision variables. Section 3 is devoted to the analytical characterization of both the value function and the optimal policy. Section 4 examines the special case of costless hedging. Section 5 gives numerical illustration in the general case.

\section{The model}

Consider a probability space $(\Omega, \mathcal{F}, \mathbb{P})$ equipped with a filtration $\left(\mathcal{F}_{t}\right)_{t \geq 0}$ which satisfies the usual conditions. Let us assume that the probability space is endowed with a standard bi dimensional Brownian motion $\left(B_{t}, B_{t}^{h}\right)$. The firm cash reserve $M_{t}$ evolves as

$$
\begin{aligned}
d M_{t} & =(r-\delta) M_{t} d t+d X_{t}-\left(\left(1-N_{t}\right)+(1+u) N_{t}\right) \psi_{t}\left(\sigma_{h} d B_{t}^{h}+p d t\right)-d Z_{t}+\alpha d R_{t} \\
& -\mathbb{1}_{\{u=1\}} a d N_{t}
\end{aligned}
$$

where the first term is the interest income, the second term is the revenue from production, the third term is the cost of hedging, the fourth term is the dividend payout, the fifth term is the cash inflow from external financing and the last term is the cost of self-financing. The dividend and issuance processes are assumed to be $\mathcal{F}_{t}$ adapted, right-continuous and non decreasing with $Z_{0}=R_{0}=0$. 
To motivate the dynamics of the cash reserves, let us describe precisely the economic environment we are considering

- Production technology

The firm uses physical capital for production. We denote by $K_{t}$ the level of capital stock at time $t$. At time 0 , the level of capital is normalized to unity. The firm's operating revenue $X_{t}$ at time $t$ is proportional to its capital stock $K_{t}$, and is given by

$$
d X_{t}=K_{t}\left(\mu d t+\sigma d B_{t}+\sigma_{h} d B_{t}^{h}\right)
$$

where $\left(B_{t}, B_{t}^{h}\right)$ is a standard bi dimensional Brownian motion.

We assume that the firm has a non predictable investment opportunity that will appear at a $\mathcal{F}_{t}$ stopping time $T$ which we assume to be independent of the Brownian motion $\left(B_{t}, B_{t}^{h}\right)$. Once the investment opportunity has come, the firm has the option to undertake it immediately or abandon it for ever. We introduce the binary control variable $u \in\{0,1\}$ to model this investment decision. If the firm decides to undertake the investment project $(u=1)$, we assume that the level of capital stock doubles. Hence, the capital stock process $K_{t}$ evolves as:

$$
K_{t}=\left(1-N_{t}\right)+(1+u) N_{t}
$$

where $N_{t}=\mathbb{1}_{\{t \geq T\}}$ is the counting process that jumps from zero to unity when the investment opportunity appears. The law of the investment appearance is assumed both to be independent of the activity in place and to be exponentially distributed with parameter $\lambda$, that is

$$
\mathbb{P}(T \leq t)=1-e^{-\lambda t}
$$

\section{- Liquidity Management}

When the firm runs out of cash, it has to either raise external funds to continue operating, or it must liquidate its assets. If the firm chooses to raise external funds, it must bear an external financing costs $1-\alpha$ proportional to the amount issued. This amounts to assume that Equation (2.1) represents the dynamics of the cash reserves up to the default time $\tau_{B}$ defined as

$$
\tau_{B}=\inf \left\{t \geq 0, M_{t}<0\right\} .
$$

We denote by $R_{t}$ the firm's cumulative external financing up to time $\mathrm{t}$ and by $Z_{t}$ the cumulative amount of dividends distributed up to time $t$.

\section{- Hedging}

Inspired by the framework proposed in [4], we assume that the firm can reduce the market risk $\sigma_{h}$ by trading in future markets. We assume that the firm can choose optimally a fraction $\psi \in[0,1]$ of the market risk $\sigma_{h}$ that is sold on future markets. We suppose that the cost of hedging is proportional to this fraction of hedged risk leading to a cost

$$
K_{t} \psi_{t} p d t
$$

where $p$ represents an additional cost per unit of hedged risk. 
- Financing the investment opportunity

When the firm decides to undertake the investment project, the firm management has to choose the optimal way to finance it. In this model, we assume that the investment opportunity entails a sunk cost $I$. The firm can self-finance an amount $a \leq I$ on its cash reserves and issue equity on the capital market to finance the difference $I-a$. We model frictions on the capital market by introducing a cost of dilution $\beta$. Even cash-rich firms may have incentives to issue equity because a self-financing investment strategy lowers the level of cash reserve and thus increases the probability to be in financial distress after investment. There is a trade-off between the cost of equity issuance to finance expansion measured by $\beta$ and the cost of recapitalization $\alpha$ in case of financial distress. The costs do not only represent the underwriting and administrative fees but also asymmetric informational cost as well. We assume that the recapitalization cost is more important than the expansion cost, namely $\alpha \leq \beta$.

We denote by $\mathcal{A}$ the set of admissible control variable $\pi=(u, Z, R, \psi, a)$ and we assume that

$$
\mathcal{A}=\left\{\pi, M_{t}^{\pi} \geq 0, e^{-r t} M_{t}^{\pi} \text { in } L^{1}, \text { and } e^{-r t} M_{t}^{\pi} \rightarrow 0 \text { p.s and in } L^{1}\right\} .
$$

Shareholders are risk-neutral and discount future cash-flows at the risk-free interest rate $r$. Let us denote by $\pi$ the set of control variables. The shareholder value function at time $t$ is given by ( see for instance [15])

$$
V_{t}=\operatorname{ess} \sup _{\pi} \mathbb{E}_{t}\left(\int_{t}^{\tau_{B}} e^{-r s}\left(d Z_{s}-d R_{s}-\frac{I-a}{\beta} \mathbb{1}_{\{u=1\}} d N_{s}\right)\right) .
$$

In our Markovian framework, $V_{t}$ is a deterministic function of both the level of cash $M_{t}$ and the indicator of the presence of the investment opportunity $N_{t}$. That is, $V_{t}=V\left(N_{t}, M_{t}\right)$ where for $n=0,1$ and $m \geq 0$,

$$
V(n, m)=\sup _{\pi \in \mathcal{A}} \mathbb{E}\left(\int_{0}^{\tau_{B}} e^{-r s}\left(d Z_{s}-d R_{s}-\frac{I-a}{\beta} \mathbb{1}_{\{u=1\}} d N_{s}\right)\right)
$$

In the absence of external financing $\operatorname{costs}(\alpha=\beta=1)$, the optimal strategy would be to distribute the initial cash reserve $M_{0}$ as dividends and to offset profits and losses by payments to or from shareholders, in other words the firm's operating revenue $X_{t}$ would coincide with $Z_{t}-R_{t}$. We call this strategy first best $(\mathrm{FB})$ in the sense that it corresponds to the maximal value that the shareholders may extract from the project see Proposition 2.1. In that case, shareholders value would be computed as follows:

- If the firm invest in the opportunity.

- when $n=1$,

$$
V_{F B}(1, m)=m+\frac{2 \mu}{r}
$$


- when $n=0$,

$$
\begin{aligned}
V_{F B}(0, m) & =m+\mathbb{E} \int_{0}^{T} e^{-r s} \mu d t+\mathbb{E} \int_{T}^{\infty} e^{-r s} 2 \mu d s-I \mathbb{E}\left(e^{-r T}\right) \\
& =m+\frac{\mu}{r}\left(1+\frac{\lambda}{r+\lambda}\right)-I \frac{\lambda}{r+\lambda} .
\end{aligned}
$$

- If the firm does not invest

$$
V_{F B}(0, m)=V_{F B}(1, m)=m+\frac{\mu}{r},
$$

As a consequence, it is optimal to invest when there is no costs of external finance if and only if $I \leq \frac{\mu}{r}$. Hereafter, we will assume

Assumption 2.1 The investment cost is lower than the expected value of the growth opportunity,

$$
I \leq \frac{\mu}{r}
$$

Next Proposition specifies the above reasoning and gives a natural upper bound for the shareholders value function.

Proposition 2.1 We have $V(n, m) \leq V_{F B}(n, m)$ for any pair $(n, m) \in\{0,1\} \times \mathbb{R}_{+}$.

Proof: Let $\pi \in \mathcal{A}$ and assume that $n=0$. We have since $M_{\tau_{B}}=0$ on $\tau_{B}<\infty$

$$
0=e^{-r \tau_{B}} M_{\tau_{B}}^{\pi}=m+\int_{0}^{\tau_{B}} e^{-r s} d M_{s}-r \int_{0}^{\tau_{B}} e^{-r s} M_{s}^{\pi} d s
$$

Using Equation (2.1), we obtain

$$
\begin{aligned}
0 & =m+\int_{0}^{\tau_{B}} e^{-r s} d X_{s}-\int_{0}^{\tau_{B}} e^{-r s}\left(\left(1-N_{s}\right)+(1+u) N_{s}\right) \psi_{s}\left(\sigma_{h} d W_{s}^{h}+p d s\right) \\
& -\int_{0}^{\tau_{B}} e^{-r s}\left(d Z_{t}-\alpha d R_{t}\right)-\int_{0}^{\tau_{B}} e^{-r s} \mathbb{1}_{\{u=1\}} a d N_{s}-\delta \int_{0}^{\tau_{B}} e^{-r s} M_{s}^{\pi} d s .
\end{aligned}
$$

Because, $M_{t}^{\pi}, \psi$ and $p$ are non negative, we get

$$
\begin{aligned}
\left(\int_{0}^{\tau_{B}} e^{-r s}\left(d Z_{s}-d R_{s}-\frac{I-a}{\beta} \mathbb{1}_{\{u=1\}} d N_{s}\right)\right) & \leq m+\int_{0}^{\tau_{B}} e^{-r s} K_{s} \mu d s+H_{\tau_{B}} \\
& -(1-\alpha) \int_{0}^{\tau_{B}} e^{-r s} d R_{s} \\
& -\left(\frac{I-a}{\beta}+a\right) \mathbb{1}_{\{u=1\}} \int_{0}^{\tau_{B}} e^{-r s} d N_{s},
\end{aligned}
$$

where

$$
H_{t}=\int_{0}^{t} e^{-r s} K_{s} \sigma d W_{s}+\int_{0}^{t} e^{-r s}\left(\left(1-N_{s}\right)+(1+u) N_{s}\right)\left(1-\psi_{s}\right) \sigma_{h} d W_{s}^{h} .
$$

Because $K_{s}$ and $\psi_{s}$ are bounded, the quadratic variation of $H$ satisfies $<H>_{\infty}<\infty$ and thus $\left(H_{t}\right)_{t \geq 0}$ is uniformly integrable. Hence, $\mathbb{E}\left(H_{\tau_{B}}\right)=0$ by the Optional sampling theorem 
([13] Theorem 3.22) .

Because $\left(R_{t}\right)_{t \geq 0}$ is increasing and $\beta \leq 1$, we get

$$
\begin{aligned}
\mathbb{E}\left(\int_{0}^{\tau_{B}} e^{-r s}\left(d Z_{s}-d R_{s}-\frac{I-a}{\beta} \mathbb{1}_{\{u=1\}} d N_{s}\right)\right) & \leq m+\mathbb{E}\left(\int_{0}^{\tau_{B}} e^{-r s} K_{s} \mu d s-I e^{-r T} \mathbb{1}_{\{u=1\}} \mathbb{1}_{\left\{T<\tau_{B}\right\}}\right) \\
& \leq m+\mathbb{E}\left(\mathbb{E}\left(\left.\int_{0}^{\tau_{B}} e^{-r s} K_{s} \mu d s\right|_{u=0}\right) \mathbb{1}_{\{u=0\}}\right) \\
& +\mathbb{E}\left(\mathbb{E}\left(\int_{0}^{\tau_{B}} e^{-r s} K_{s} \mu d s-\left.I e^{-r T}\right|_{u=1}\right) \mathbb{1}_{\{u=1\}}\right) .
\end{aligned}
$$

Now,

$$
\mathbb{E}\left(\left.\int_{0}^{\tau_{B}} e^{-r s} K_{s} \mu d s\right|_{u=0}\right) \leq \frac{\mu}{r}
$$

and

$$
\mathbb{E}\left(\int_{0}^{\tau_{B}} e^{-r s} K_{s} \mu d s-\left.I e^{-r T}\right|_{u=1}\right) \leq \frac{\mu}{r}\left(1+\frac{\lambda}{r+\lambda}\right)-I \frac{\lambda}{r+\lambda} .
$$

Therefore,

$$
\begin{aligned}
\mathbb{E}\left(\int_{0}^{\tau_{B}} e^{-r s}\left(d Z_{s}-d R_{s}-\frac{I-a}{\beta} \mathbb{1}_{\{u=1\}} d N_{s}\right)\right) & \leq \max \left(m+\frac{\mu}{r}, m+\frac{\mu}{r}\left(1+\frac{\lambda}{r+\lambda}\right)-I \frac{\lambda}{r+\lambda}\right) \\
& =V_{F B}(0, m) .
\end{aligned}
$$

\section{Analytical Characterization of the optimal policy of the firm}

In order to compute the two value functions $V(0, m)$ and $V(1, m)$, we will proceed recursively with respect to the arrival date of the investment opportunity $T$.

\subsection{After the arrival date of the investment opportunity}

Let us first assume that the investment opportunity has already occurred, that is $t \geq T$ or equivalently $N_{t}=1$. At date $T$, either the firm has undertaken the investment $(u=1)$ or the firm has given up the investment opportunity $(u=0)$. Let us characterize the value function associated to each situation.

Assume first that the investment opportunity has not been undertaken at time $T$ or equivalently $u=0$. Therefore, cash reserves evolve as

$$
\begin{aligned}
d M_{t} & =(r-\delta) M_{t} d t+\mu d t+\sigma d B_{t}+\sigma_{h} B_{t}^{h}-\psi_{t}\left(\sigma_{h} B_{t}^{h}+p d t\right) \\
& -d Z_{t}+\alpha d R_{t} .
\end{aligned}
$$

Denote by $W^{0}$ the shareholders value function associated to this scenario. That is

$$
W^{0}(m)=\sup _{\pi} \mathbb{E}\left(\int_{0}^{\tau_{B}} e^{-r s}\left(d Z_{s}-d R_{s}\right)\right)
$$


where the cash reserves process $M_{t}$ follows (3.2) with $M_{0}=m$.

Next proposition gives some results about the dependence of the value function with respect to both hedging cost $p$ and profitability $\mu$.

Proposition 3.2 The function $W^{0}$ is a decreasing function of $p$ and an increasing function of $\mu$.

Proof: Let $p>p_{0}$. When the hedging cost is $p_{0}$, the cash reserves evolve as

$$
\begin{aligned}
d M_{t} & =(r-\delta) M_{t} d t+\mu d t+\sigma d B_{t}+\sigma_{h} B_{t}^{h}-\psi_{t}\left(\sigma_{h} B_{t}^{h}+p_{0} d t\right) \\
& -d Z_{t}+\alpha d R_{t} .
\end{aligned}
$$

We write $W^{0}(m, p)$ to highlight the dependence of the value function with respect to the hedging cost p.

Let $\left(Z_{t}^{\varepsilon}, R_{t}^{\varepsilon}, \psi^{\varepsilon}\right)$ an $\varepsilon$-optimal strategy for $W^{0}$ when the hedging cost is $p$ and insert the policy $\left(Z_{t}^{\varepsilon}+\int_{0}^{t} \psi_{s}^{\varepsilon}\left(p-p_{0}\right) d s, R_{t}^{\varepsilon}, \psi^{\varepsilon}\right)$ in equation (3.4) to obtain

$$
\begin{aligned}
W^{0}\left(m, p_{0}\right) & \geq W^{0}(m, p)-\varepsilon+\mathbb{E}\left(\int_{0}^{\tau_{B}} e^{-r s} \psi_{s}^{\varepsilon}\left(p-p_{0}\right) d s\right) \\
& \geq W^{0}(m, p)-\varepsilon
\end{aligned}
$$

for arbitrarily $\varepsilon$.

The proof of the behavior of $W^{0}$ with respect to the profitability $\mu$ is similar and thus omitted.

The analytical characterization in terms of Hamilton-Jacobi-Bellman equations of mixed singular/regular control problems is now well established (see for instance Fleming and Soner [9]) and we expect that the value function $W^{0}$ be a solution of the free boundary problem

$$
\max \left(\max _{\psi \in(0,1)} L(\psi) W, 1-W_{m}, W_{m}-\frac{1}{\alpha}\right)=0
$$

where

$$
L(\psi) W=\left\{\left(\frac{\sigma^{2}+(1-\psi)^{2} \sigma_{h}^{2}}{2}\right) W_{m m}+[(r-\delta) m+\mu-\psi p] W_{m}-r W\right\}
$$

However, it is difficult in general to prove that there is an unique solution to (3.5) in a classical sense. In order to prove that $W^{0}$ is a solution of (3.5), we have to use economic intuition. Costly external finance leads shareholders to accumulate cash reserves in order to reduce the risk of bearing these costs. However, the marginal value of these reserves is likely to decrease (as the level of reserves increases) since external financing then becomes less likely. This speaks for a concave value function. The concavity of the value function, together with a marginal value bounded below by one, yields that shareholders will distribute dividends when the marginal value of the firm is exactly one. Therefore, we claim the existence of a threshold $b_{0}^{*}$ above which the firm distributes all the surplus as dividends. 
Based on [6], it is moreover reasonable to think that equity will be issued only when the cash reserves are depleted. This means that we are looking for a pair $\left(W^{0}, b_{0}^{*}\right)$ such that

$$
\max _{\psi \in(0,1]} L(\psi) W^{0}=0 \text { for } m \leq b_{0}^{*}
$$

and

$$
W_{m}^{0}\left(b_{0}^{*}\right)=1, W_{m m}^{0}\left(b_{0}^{*}\right)=0 \text { and } W_{m}^{0}(0)=\frac{1}{\alpha} .
$$

The following verification theorem establishes the desired result.

Proposition 3.3 Assume there exists a twice continuously differentiable concave function $W$ and $a$ constant $b_{0}^{*}$ such that

$$
\begin{gathered}
\forall m \leq b_{0}^{*} \quad \max _{\psi \in(0,1]} L(\psi) W=0 \text { and } W_{m}(m) \geq 1, W_{m}(m) \leq \frac{1}{\alpha} \\
\forall m \geq b_{0}^{*} \quad W_{m}(m)=1 \text { and } \max _{\psi \in(0,1]} L(\psi) W \leq 0
\end{gathered}
$$

together with the initial condition:

$$
\max \left(-W(0), \frac{1}{\alpha}-W_{m}(0)\right)=0
$$

then $W=W^{0}$.

Proof: Fix a policy $\pi \in \mathcal{A}$. According to Proposition 2.1, we may assume that the random variable $\int_{0}^{\tau_{B}} e^{-r s}\left(d Z_{s}-d R_{s}\right)$ is in $L^{1}$. Let us write the processes $Z_{t}=Z_{t}^{c}+Z_{t}^{d}$ and $R_{t}=R_{t}^{c}+R_{t}^{d}$ where $Z_{t}^{c}$ (resp. $R_{t}^{c}$ ) are the continuous part of $Z_{t}$ (resp. $R_{t}$ ) and $Z_{t}^{d}$ (resp. $\left.R_{t}^{d}\right)$ are the pure discontinuous part of $Z_{t}$ (resp. $R_{t}$ ). Let:

$$
\begin{aligned}
d M_{t} & =(r-\delta) M_{t} d t+\mu d t+\sigma d B_{t}+\sigma_{h} d B_{t}^{h}-\psi_{t}\left(\sigma_{h} d B_{t}^{h}+p d t\right) \\
& -d Z_{t}+\alpha d R_{t} \\
M_{0} & =m
\end{aligned}
$$

be the dynamic of cash reserves under the policy $\left(\psi_{t}, Z_{t}, R_{t}\right)$. Using the generalized Itô formula (see Dellacherie and Meyer Theorem VIII.27), we can write:

$$
\begin{aligned}
e^{-r\left(t \wedge \tau_{B}\right)} W\left(M_{\left(t \wedge \tau_{B}\right)}\right) & =W(m)+\int_{0}^{\left(t \wedge \tau_{B}\right)} e^{-r s} L\left(\psi_{s}\right) W\left(M_{s}\right) d s \\
& +\int_{0}^{\left(t \wedge \tau_{B}\right)} e^{-r s} W_{m}\left(M_{s}\right)\left(\sigma d B_{t}+\sigma_{h} d B_{t}^{h}\right)-\int_{0}^{\left(t \wedge \tau_{B}\right)} e^{-r s} W_{m}\left(M_{s}\right) d Z_{s}^{c} \\
& +\int_{0}^{\left(t \wedge \tau_{B}\right)} e^{-r s} W_{m}\left(M_{s}\right) \alpha d R_{s}^{c}+\sum_{s \leq\left(t \wedge \tau_{B}\right)} e^{-r s}\left(W\left(M_{s}\right)-W\left(M_{s-}\right)\right),
\end{aligned}
$$

Because $W$ satisfies (3.7) and (3.8) the second term of the right hand side is negative. Because $W_{m}$ is bounded, $\left(1 \leq W_{m}(m) \leq \frac{1}{\alpha}\right)$ the third term is a centered square integrable 
martingale. Taking expectations, we get

$$
\begin{aligned}
E\left(e^{-r\left(t \wedge \tau_{B}\right)} W\left(M_{t \wedge \tau_{B}}\right)\right) & \leq W(m)-E \int_{0}^{t \wedge \tau_{B}} e^{-r s} W_{m}\left(M_{s}\right) d Z_{s} \\
& +E \int_{0}^{t \wedge \tau_{B}} e^{-r s} W_{m}\left(M_{s}\right) \alpha d R_{s} \\
& +E \sum_{s \leq t \wedge \tau_{B}} e^{-r s}\left(W\left(M_{s}\right)-W\left(M_{s-}\right)-W_{m}\left(M_{s-}\right)\left(M_{s}-M_{s-}\right)\right) .
\end{aligned}
$$

By concavity $W\left(M_{s}\right)-W\left(M_{s-}\right)-W_{m}\left(M_{s-}\right)\left(M_{s}-M_{s-}\right)<0$. Therefore, using the fact that $-W_{m} \geq-\frac{1}{\alpha}$ and $W_{m} \geq 1$,

$$
W(m) \geq E\left(e^{-r\left(t \wedge \tau_{B}\right)} W\left(M_{t \wedge \tau_{B}}\right)\right)+E \int_{0}^{t \wedge \tau_{B}} e^{-r s}\left(d Z_{s}-d R_{s}\right) .
$$

In order to end the proof, we have to get rid of the first-term of the right-hand side. But,

$$
E\left(e^{-r\left(t \wedge \tau_{B}\right)} W\left(M_{t \wedge \tau_{B}}\right)\right)=E\left(e^{-r \tau_{B}} W(0) \mathbb{1}_{\left\{\tau_{B}<t\right\}}\right)+E\left(e^{-r t} W\left(M_{t}\right) \mathbb{1}_{\left\{\tau_{B}>t\right\}}\right) .
$$

Now, $W(m) \leq W(0)+W_{m}(0) m$ by concavity therefore

$$
E\left(e^{-r t} W\left(M_{t}\right) \mathbb{1}_{\left\{\tau_{B}>t\right\}}\right) \leq W(0) e^{-r t}+W_{m}(0) E\left(e^{-r t} M_{t}\right) .
$$

Let $t$ tend to $\infty$ to get (because $\lim _{t \rightarrow \infty} E\left(e^{-r t} M_{t}\right)=0$ for $\pi \in \mathcal{A}$ )

$$
\begin{aligned}
W(m) & \geq E\left(e^{-r \tau_{B}} W(0) \mathbb{1}_{\left\{\tau_{B}<\infty\right\}}\right)+E \int_{0}^{\tau_{B}} e^{-r s}\left(d Z_{s}-d R_{s}\right) \\
& \geq E \int_{0}^{\tau_{B}} e^{-r s}\left(d Z_{s}-d R_{s}\right) \text { because } W(0) \geq 0 .
\end{aligned}
$$

The reverse inequality comes from the fact that the solution $W$ of (3.7)-(3.9) is attainable by an admissible strategy. Nevertheless, we have to distinguish the two cases $W(0)=0$ and $W_{m}(0)=\frac{1}{\alpha}$. Because the associated proofs both rely on Skohorod lemma and are thus quite similar for the two cases, we only focus on the case $W_{m}(0)=\frac{1}{\alpha}$.

Let $\psi_{t}^{*}$ be the maximizer in (3.7), and let $\left(M_{t}^{*}, Z_{t}^{*}, R_{t}^{*}\right)$ be the solution of

$\left.M_{t}^{*}=m+\int_{0}^{t}(r-\delta) M_{s} d s+\mu d s+\sigma d W_{s}+\sigma_{h} d W_{s}^{h}-\psi_{s}^{*}\left(\sigma_{h} d W_{s}^{h}+p d s\right)+\alpha R_{t}^{*}-Z_{t}^{*} 3.10\right)$

where

$$
Z_{t}^{*}=\int_{0}^{t} \mathbb{1}_{M_{s}^{*}=b_{0}^{*}} d Z_{s}^{*}
$$

and

$$
R_{t}^{*}=\int_{0}^{t} \mathbb{1}_{M_{s}^{*}=0} d R_{s}^{*}
$$

whose existence is guaranteed by standard results on the Skorokhod problem. The strategy $\pi^{*}=\left(Z_{t}^{*}, R_{t}^{*}, \psi^{*}, \infty\right)$ is admissible because $M_{t}^{*}=M_{t}^{\pi^{*}}$ is bounded due to Equation (3.10). 
Using generalized Itô formula again and noting that the process $\left(M_{t}^{*}\right)_{t \geq 0}$ is continuous, we obtain for $m \in\left[0, b_{0}^{*}\right]$

$$
\begin{aligned}
e^{-r t} W\left(M_{t}^{*}\right) & =W(m)+\int_{0}^{t} e^{-r s} L\left(\psi_{s}^{*}\right) W\left(M_{s}^{*}\right) d s \\
& +\int_{0}^{t} e^{-r s} W_{m}\left(M_{s}^{*}\right)\left(\sigma d W_{s}+\sigma_{h} d W_{s}^{h}\right)-\int_{0}^{t} e^{-r s} W_{m}\left(M_{s}^{*}\right) d Z_{s}^{*} \\
& +\int_{0}^{t} e^{-r s} W_{m}\left(M_{s}^{*}\right) \alpha d R_{s}^{*}
\end{aligned}
$$

Because $M_{t}^{*} \in\left[0, b_{0}^{*}\right]$, the second term vanishes because $L\left(\psi_{s}^{*}\right) W(m)=0$ on $\left(0, b_{0}^{*}\right)$. Because $W_{m}$ is bounded, the stochastic integral is a martingale and therefore we get after taking expectations

$$
\begin{aligned}
E\left(e^{-r t} W\left(M_{t}^{*}\right)\right) & =W(m)-E \int_{0}^{t} e^{-r s} W_{m}\left(b_{0}^{*}\right) d Z_{s}^{*} \\
& +E \int_{0}^{t} e^{-r s} W_{m}(0) \alpha d R_{s}^{*} .
\end{aligned}
$$

Using $W_{m}(0)=\frac{1}{\alpha}$, we have

$$
W(m)=E \int_{0}^{t} e^{-r s}\left(W_{m}\left(b_{0}^{*}\right) d Z_{s}^{*}-d R_{s}^{*}\right)+E\left(e^{-r t} W\left(M_{t}^{*}\right)\right) .
$$

Let $t$ tend to $+\infty$ to conclude by noting again that $W_{m}\left(b_{0}^{*}\right)=1, W\left(M_{t}^{*}\right)$ is bounded by $W\left(b_{0}^{*}\right)$.

Remark 3.1 Note that the optimal issuance policy is the same as in [6] . Either, it is optimal to never issue equity and to default the first time the cash reserves hit zero or it is optimal to issue equity at each time the cash reserves hit zero. For the latter case, the firm will never default. Nevertheless, the optimal equity issuance will not depend only on the level of issuance cost $\alpha$ but also on the level of hedging cost $p$.

We will now focus on the existence of a pair $\left(W, b_{0}^{*}\right)$ that satisfies Proposition 3.3. We first note that the operator $L(\psi)$ can be decomposed as follows:

$$
L(\psi) W(m)=L(0) W(m)-\psi\left(\sigma_{h}^{2} W_{m m}(m)\left(1-\frac{\psi}{2}\right)+p W_{m}(m)\right) .
$$

Therefore, an optimal hedging is a maximization (assuming that $W$ is concave) of the parabola

$$
-\psi\left(\sigma_{h}^{2} W_{m m}(m)\left(1-\frac{\psi}{2}\right)+p W_{m}(m)\right) .
$$

The first order condition gives

$$
\psi^{*}(m)=1+\frac{p}{\sigma_{h}^{2}} \frac{W_{m}}{W_{m m}}(m) .
$$

Two cases have to be considered: 
1. $\sigma_{h}^{2} W_{m m}+p W_{m} \geq 0$. In that case the maximum of the parabola is below 0 so $L(0) W \geq L(\psi) W$ for every $\psi \in[0,1]$,

2. $\sigma_{h}^{2} W_{m m}+p W_{m}<0$. and the maximum of the parabola is in $[0,1]$ assuming $W$ is concave. Due to the concavity of $W$ along with $W_{m} \geq 1$, the optimal level of hedging $\psi^{*}(m)$ is strictly lower than one. Therefore, it is never optimal to fully hedge except when $p=0$.

We make the following guess about the features of the value function: $W^{0}$ is a concave twice continuously differentiable function which implies since $W_{m m}^{0}\left(b_{0}^{*}\right)=0$ that there is some $0 \leq \tilde{b}_{0}<b_{0}^{*}$ such that $\psi^{*}\left(\tilde{b}_{0}\right)=0$ and $\psi^{*}=0$ on the interval $\left(\tilde{b}_{0}, b_{0}^{*}\right)$. As a consequence, we can split the computation of the value function in two stages. First, solve on $\left(\tilde{b}_{0}, b_{0}^{*}\right)$

$$
\left(\frac{\sigma^{2}+\sigma_{h}^{2}}{2}\right)(W)_{m m}+[(r-\delta) m+\mu] W_{m}-r W=0
$$

under the boundary conditions

$$
W_{m}\left(b_{0}^{*}\right)=1,(W)_{m m}\left(b_{0}^{*}\right)=0,
$$

and secondly, solve on $\left(0, \tilde{b}_{0}\right)$ the non-linear ordinary differential equation

$$
\frac{\sigma^{2}}{2}(W)_{m m}+[(r-\delta) m+\mu-p] W_{m}-r W-\frac{p^{2}}{2 \sigma_{h}^{2}} \frac{W_{m}^{2}}{W_{m m}}(m)=0
$$

with the boundary condition $W(0)=0$ or $W_{m}(0)=\frac{1}{\alpha}$.

Note that it could happen that $\tilde{b}_{0}=0$. In that case, the optimal liquidity management policy coincides with the one described in [6].

In order to prove the existence of a concave solution satisfying Proposition 3.3, we have to introduce the following functions. Fix $b \geq 0$ and denote by $V^{b}$ the concave (see [6]) solution of

$$
L(0) V^{b}=0 \text { with } V_{m}^{b}(b)=1, V_{m m}^{b}(b)=0 .
$$

According to Lemma A.2 in [6], $V^{b}(0)$ is a strictly decreasing function of $b$ while the first derivative $V_{m}^{b}(0)$ is a strictly increasing function of $b$. Because, $\lim _{b \rightarrow 0} V^{b}(0)=\frac{\mu}{r}$ and $\lim _{b \rightarrow+\infty} V^{b}(0)=-\infty$ (see the Internet Appendix of [6]), there exists $b_{0}$ such that $V^{b_{0}}(0)=0$. Next proposition characterizes the shareholders value function by proving the existence of a concave solution denoted abusively $W^{0}$ of Proposition 3.3. More precisely,

Proposition 3.4 There exist a concave twice continuously differentiable function $W^{0}$ and a threshold $b_{0}^{*}$ that satisfy the assumptions of Proposition 3.3.

Proof: We find it helpful to organize the proof in two cases.

First Case: Assume that $V_{m}^{b_{0}}(0) \geq \frac{1}{\alpha}$. According to Lemma A.2 in [6], there is some $b_{1}>0$ such that $V_{m}^{b_{1}}(0)=\frac{1}{\alpha}$. Moreover, the hypothesis $V_{m}^{b_{0}}(0) \geq \frac{1}{\alpha}$ implies $b_{1} \leq b_{0}$ and thus $V^{b_{1}}(0)>0$. Let us define

$$
\theta^{b_{1}}(m)=\sigma_{h}^{2} V_{m m}^{b_{1}}(m)+p V_{m}^{b_{1}}(m)
$$


The function $\frac{V_{m}^{b_{1}}(m)}{V_{m m}^{b_{1}}(m)}$ is decreasing because the sign of the derivative of $\frac{V_{m}^{b_{1}}(m)}{V_{m m}^{b_{1}}(m)}$ is given by the sign of

$$
\frac{\sigma^{2}+\sigma_{h}^{2}}{2}\left(\left(V_{m m}^{b_{1}}(m)\right)^{2}-V_{m}^{b_{1}}(m) V_{m m m}^{b_{1}}(m)\right)=-\delta\left(V_{m}^{b_{1}}(m)\right)^{2}+r V_{m m}^{b_{1}}(m) V^{b_{1}}(m)<0 .
$$

Therefore, there is at most a threshold $\tilde{m}_{1}<b_{1}$ such that $\theta^{b_{1}}\left(\tilde{m}_{1}\right)=0$. The existence of $\tilde{m}_{1}$ is given by the sign of $\theta^{b_{1}}(0)$ since $\theta^{b_{1}}\left(b_{1}\right)=p \geq 0$. Thus, if the hedging cost $p$ is such that

$$
\sigma_{h}^{2} V_{m m}^{b_{1}}(0)+\frac{p}{\alpha} \geq 0
$$

then it is optimal to never hedge and the pair $\left(V^{b_{1}}, b_{1}\right)$ satisfies Proposition 3.3.

Note that

$$
\theta^{b}(m)=\frac{2 \sigma_{h}^{2}}{\sigma_{h}^{2}+\sigma^{2}} r V^{b}(0)+\left(p-\frac{2 \sigma_{h}^{2} \mu}{\sigma_{h}^{2}+\sigma^{2}}\right) V_{m}^{b}(0) .
$$

A sufficient condition on the hedging cost $p$ to have (3.12) is thus

$$
p \geq \frac{2 \sigma_{h}^{2} \mu}{\sigma_{h}^{2}+\sigma^{2}} .
$$

Assume now that $p$ is such that (3.12) does not hold and note $\tilde{m}_{1}$ the solution of $\theta^{b_{1}}(m)=0$. We are interested now by the existence and the uniqueness of a concave solution to the non linear O.D.E. on $\left(0, \tilde{m}_{1}\right)$

$$
\frac{\sigma^{2}}{2} W_{m m}+[(r-\delta) m+\mu-p] W_{m}-r W-\frac{p^{2}}{2 \sigma_{h}^{2}} \frac{W_{m}^{2}}{W_{m m}}(m)=0
$$

with $W\left(\tilde{m}_{1}\right)=V^{b_{1}}\left(\tilde{m}_{1}\right)$ and $W_{m}\left(\tilde{m}_{1}\right)=V_{m}^{b_{1}}\left(\tilde{m}_{1}\right)$. We first notice that if such a concave solution $W^{0}$ exists then $W_{m m}^{0}\left(\tilde{m}_{1}\right)=V_{m m}^{b_{1}}\left(\tilde{m}_{1}\right)$ and thus $W^{0}$ is locally strictly concave in a left neighborhood of $\tilde{m}_{1}$. In order to see this, we let $m$ tend to $\tilde{m}_{1}$ in Equation (3.14) and use the continuous and smooth fit at $\tilde{m}_{1}$ to get

$$
0=\frac{p W_{m}^{0}\left(\tilde{m}_{1}\right)}{2}\left(1-\frac{V_{m m}^{b_{1}}\left(\tilde{m}_{1}\right)}{W_{m m}^{0}\left(\tilde{m}_{1}\right)}\right)+\frac{\sigma^{2}}{2}\left(V_{m m}^{b_{1}}\left(\tilde{m}_{1}\right)-W_{m m}^{0}\left(\tilde{m}_{1}\right)\right) .
$$

Thus the local concavity of $W^{0}$ implies the continuity of $W_{m m}^{0}$ at $\tilde{m}_{1}$. If we multiply (3.14) by $W_{m m}^{0}(m)$, we see that $W_{m m}^{0}$ is the root of a second order polynomial equation and thus for every $m \geq 0$

$$
W_{m m}^{0}(m)=\frac{r W^{0}(m)-((r-\delta) m+\mu-p) W_{m}^{0}(m)+\varepsilon(m) \sqrt{\Delta\left[W^{0}\right](m)}}{\sigma^{2}}
$$

where $\varepsilon(m)=+1$ or -1 and

$$
\left.\Delta[W](m)=((r-\delta) m+\mu-p) W_{m}(m)-r W(m)\right)^{2}+\sigma^{2} \frac{p^{2}}{\sigma_{h}^{2}}\left(W_{m}(m)\right)^{2} .
$$

Let's take the concave solution satisfying $\varepsilon(m)=-1$. We finally obtain the existence and uniqueness of a concave solution $W^{0}$ of $(3.14)$ on $\left(0, \tilde{m}_{1}\right)$ with continuous and smooth-fit 
at $\tilde{m}_{1}$ by the Cauchy-Lipschitz Theorem.

At this stage, for every fixed $b$, we have the existence and the uniqueness of a concave and twice continuously differentiable function $W^{b}$ solution of

$$
L(0) W^{b}=0 \text { on }(\tilde{m}(b), b)
$$

and

$$
L\left(\psi^{*}\right) W^{b}=0 \text { on }(0, \tilde{m}(b))
$$

with $W_{m}^{b}(b)=1, W_{m m}^{b}(b)=0$ and $\tilde{m}(b)$ is the root of $\sigma_{h}^{2} W_{m m}^{b}(m)+p W_{m}^{b}(m)$. We must now check that there is some $b_{0}^{*}$ such that $W_{m}^{b_{0}^{*}}(0)=\frac{1}{\alpha}, W^{b_{0}^{*}}(0) \geq 0$ and prove that $\psi^{*}$ remains in $[0,1]$ on $\left[0, \tilde{m}\left(b_{0}^{*}\right)\right]$.

To see this, let us denote $k=V^{b_{1}}-W^{b_{1}}$ and remember that $\tilde{m}_{1}=\tilde{m}\left(b_{1}\right)$. Similarly to Equation (3.11) we introduce

$$
\Theta^{b_{1}}(m)=\sigma_{h}^{2} W_{m m}^{b_{1}}(m)+p W_{m}^{b_{1}}(m)
$$

A straightforward computation yields that

$$
L(0) k(m)=-\frac{\left(\Theta^{b_{1}}\right)^{2}(m)}{2 \sigma_{h}^{2} W_{m m}^{b_{1}}(m)}
$$

on $\left(0, \tilde{m}_{1}\right)$ where $\Theta^{b_{1}}$ is given by Equation (3.18). Therefore, $L(0) k \geq 0$ on $\left(0, b_{1}\right)$ since $V^{b_{1}}$ and $W^{b_{1}}$ coincide on $\left(\tilde{m}_{1}, b_{1}\right)$ with $k\left(\tilde{m}_{1}\right)=k_{m}\left(\tilde{m}_{1}\right)=k_{m m}\left(\tilde{m}_{1}\right)=0$. Let us differentiate $L(0) k(m)$ on $\left(0, \tilde{m}_{1}\right)$ to get

$$
\left(\frac{\sigma^{2}+\sigma_{h}^{2}}{2}\right) k_{m m m}+(\mu+(r-\delta) m) k_{m m}-\delta k_{m}=-\left(\frac{\Theta^{b_{1}} \Theta_{m}^{b_{1}}}{\sigma_{h}^{2} W_{m m}^{b_{1}}}-\frac{\left(\Theta^{b_{1}}\right)^{2} W_{m m m}^{b_{1}}}{2 \sigma_{h}^{2}\left(W_{m m}^{b_{1}}\right)^{2}}\right) .
$$

Using smooth-fit at $\tilde{m}_{1}$ and $\Theta^{b_{1}}\left(\tilde{m}_{1}\right)=0$, we get $k_{m m m}\left(\tilde{m}_{1}\right)=0$. Differentiating one more time and letting $m$ to $\tilde{m}_{1}$, we get

$$
\left(\frac{\sigma^{2}+\sigma_{h}^{2}}{2}\right) k_{(4)}\left(\tilde{m}_{1}\right)=\frac{-\left(\Theta_{m}^{b_{1}}\right)^{2}}{\sigma_{h}^{2} W_{m m}^{b_{1}}} \geq 0 .
$$

Therefore, $k$ is a convex positive function in a left neighborhood of $\tilde{m}_{1}$. We will prove that the first derivative $k_{m}$ is always non positive. Assume the contrary and denote by $m_{0}$ the highest level of cash such that $k_{m}\left(m_{0}\right)=0$. Note that $k$ must be concave at $m_{0}$ and thus

$$
L(0) k\left(m_{0}\right)=\left(\frac{\sigma^{2}+\sigma_{h}^{2}}{2}\right) k_{m m}\left(m_{0}\right)-r k\left(m_{0}\right)<0
$$

which contradicts $L(0) k \geq 0$. Hence, $W_{m}^{b_{1}}(0) \geq \frac{1}{\alpha}$.

Set $Y(m)=\left(W(m), W_{m}(m)\right)$ such that $Y_{m}=F(m, Y)$ with $F$ locally Lipschitz with respect to $Y$. Denote by $y_{b}=\left(\frac{\mu+(r-\delta) b}{r}, 1\right)$ and $\phi(m, b)$ the unique solution of the o.d.e such that $\phi(b, b)=y_{b}$. By Cauchy-Lipschitz Theorem, the function $\phi$ is jointly continuous and thus there is a $b_{0}^{*} \leq b_{1}$ such that $W_{m}^{b_{0}^{*}}(0)=\frac{1}{\alpha}$.

Besides we know that $W^{b_{0}^{*}}$ is above $V^{b_{1}}$ because $V^{b_{1}}$ corresponds to an admissible hedging 
strategy, thus $W^{b_{0}^{*}}>0$.

We then decide to set $W^{0}=W^{b_{0}^{*}}>0$. In order to end the proof, we need to check that the strategy $\psi^{*}$ is admissible, that is $\psi^{*}$ is in $[0,1]$ on $\left[0, \tilde{m}\left(b_{0}^{*}\right)\right]$. The solution being concave we know that $\psi^{*}<1$ on $\left[0, \tilde{m}\left(b_{0}^{*}\right)\right]$.

Using the fact that

$$
\left(\frac{\sigma^{2}}{2}+\frac{p^{2}}{2 \sigma_{h}^{2}} \frac{\left(W_{m}^{0}\right)^{2}}{\left(W_{m m}^{0}\right)^{2}}\right) W_{m m m}^{0}=\left(\frac{p^{2}}{\sigma_{h}^{2}}+\delta\right) W_{m}^{0}-((r-\delta) m+\mu-p) W_{m m}^{0},
$$

the sign of the first derivative of $\psi^{*}$ is given by the sign of

$$
\frac{\sigma^{2}}{2}\left(\left(W_{m m}^{0}\right)^{2}-W_{m}^{0} W_{m m m}^{0}\right)=-\left(W_{m}^{0}\right)^{2} \delta+r W^{0} W_{m m}^{0}
$$

which is nonpositive because $W^{0}$ is a positive concave function.

We conclude that $\psi^{*}$ is under 1 , decreasing to 0 on $\left[0, \tilde{m}\left(b_{0}^{*}\right)\right]$ and thus $\psi^{*}$ satisfies the constraints. As a consequence, the existence of $W^{0}$ has been proved for the set of parameters for which it is optimal to issue equity when the cash reserves are depleted.

Second Case: Assume $V_{m}^{b_{0}}(0)<\frac{1}{\alpha}$. In this case $V^{b_{1}}(0)<0$. The previous arguments can be used by replacing $V^{b_{1}}$ by $V^{b_{0}}$ to build $W^{b_{0}}$ solution to problem (3.16-3.17). Furthermore, we get that $W_{m}^{b_{0}}(0) \geq V_{m}^{b_{0}}(0)$.

Two cases must be distinguished.

- First, there exists a threshold $b_{0}^{*} \leq b_{0}$ such that $W_{m}^{b_{0}^{*}}(0)=\frac{1}{\alpha}$. Therefore, the function $W^{b_{0}^{*}}$ satisfies Proposition 3.3 and thus $W^{0}=W^{b_{0}^{*}}$. This is an interesting case since the possibility of hedging makes profitable equity issuance, whereas without the possibility of hedging equity issuance is too costly to prevent the firm from going bankrupt.

- Assume now there is no threshold $b \leq b_{0}$ such that $W_{m}^{b}(0)=\frac{1}{\alpha}$. We will prove this implies $\max _{b} W_{m}^{b}(0)<\frac{1}{\alpha}$. To see this, suppose, reasoning by contradiction that there is some $b>b_{0}$ such that $W_{m}^{b}(0)=\frac{1}{\alpha}$ and $W_{m}^{b}(0)>0$. By concavity and because $W^{b}(b)<V^{b_{0}}\left(b_{0}\right)+\left(b-b_{0}\right)$, we have $W_{m}^{b}\left(b_{0}\right)>1$ and $W^{b}\left(b_{0}\right)<V^{b_{0}}\left(b_{0}\right)$. Introducting $k^{b}=V^{b_{0}}-W_{m}^{b}$, we have $k^{b}(0)<0, k_{m}^{b}(0)<0, k^{b}\left(b_{0}\right)>0, k_{m}^{b}\left(b_{0}\right)<0$ and as previously shown $L(0) k^{b}(m)=-\frac{\left(\Theta^{b}\right)^{2}(m)}{2 \sigma_{h}^{2} W_{m m}^{b}(m)} \geq 0$ on $(0, \tilde{m}(b)), L(0) k^{b}(m)=0$ on $\left(\tilde{m}(b), b_{0}\right)$ if $\tilde{m}(b) \leq b_{0}$. Applying the maximum principle on $\left(0, b_{0}\right)$, we obtain a contradiction.

Thus, it is never optimal in that case to issue equity when the cash reserve are depleted regardless of the hedging policy. To conclude, we have to prove the existence of a function $W^{b}$ solution to problem (3.16-3.17) with $W^{b}(0)=0$.

To do this, let us take $b=b_{0}+\frac{\mu}{\delta}$. By concavity of $W^{b}, k^{b}\left(b_{0}\right) \geq \frac{\mu}{r}$. Using the maximal principle between 0 and $b_{0}$ we get $k^{b}(0) \geq k^{b}\left(b_{0}\right)$ and therefore $\frac{\mu}{r}-W^{b}(0) \geq k^{b}\left(b_{0}\right) \geq \frac{\mu}{r}$ and $W^{b}(0) \geq 0$. By continuity there exits $b_{1}$ such that $k^{b_{1}}=0$ or equivalently $W^{b_{1}}(0)=0$. Finally, the arguments to prove that $\psi^{*}$ is in $[0,1]$ are the same. 
Corollary 3.1 The optimal level of cash $b_{0}^{*}$ is an increasing function of the hedging cost $p$.

Proof: Let $p>p_{0}$. We write $W^{0}(m, p)$ to highlight the dependence of the value function with respect to the hedging cost $p$. By continuity, we have

$$
W^{0}\left(b_{0}^{*}(p), p\right)-W^{0}\left(b_{0}^{*}\left(p_{0}\right), p_{0}\right)=\left(1-\frac{\delta}{r}\right)\left(b_{0}^{*}(p)-b_{0}^{*}\left(p_{0}\right)\right) .
$$

But using Proposition 3.2, we have

$$
\begin{aligned}
W^{0}\left(b_{0}^{*}(p), p\right)-W^{0}\left(b_{0}^{*}\left(p_{0}\right), p_{0}\right) & =W^{0}\left(b_{0}^{*}(p), p\right)-W^{0}\left(b_{0}^{*}(p), p_{0}\right)+W^{0}\left(b_{0}^{*}(p), p_{0}\right)-W^{0}\left(b_{0}^{*}\left(p_{0}\right), p_{0}\right) \\
& \leq W^{0}\left(b_{0}^{*}(p), p_{0}\right)-W^{0}\left(b_{0}^{*}\left(p_{0}\right), p_{0}\right) .
\end{aligned}
$$

Using the concavity of $W^{0}$ and the smooth-fit at $b_{0}^{*}\left(p_{0}\right)$ we get $W^{0}\left(b_{0}^{*}(p), p_{0}\right)-W^{0}\left(b_{0}^{*}\left(p_{0}\right), p_{0}\right) \leq$ $b_{0}^{*}(p)-b_{0}^{*}\left(p_{0}\right)$. Consequently,

$$
\frac{\delta}{r}\left(b_{0}^{*}(p)-b_{0}^{*}\left(p_{0}\right)\right) \geq 0
$$

Next Corollary gives a sufficient condition to ensure that the no-hedging policy is optimal, namely $W^{0}=V^{b_{0}}$.

Corollary 3.2 Assume that $V_{m}^{b_{0}}(0) \leq \frac{1}{\alpha}$ and $p \geq \frac{2 \sigma_{h}^{2} \mu}{\sigma_{h}^{2}+\sigma^{2}}$. Then, the value function $W^{0}$ coincides with $V^{b_{0}}$.

Proof: Using Equation (3.13), the assumption $p \geq \frac{2 \sigma_{h}^{2} \mu}{\sigma_{h}^{2}+\sigma^{2}}$ implies that $\theta^{b_{0}}(0) \geq 0$ because $V^{b_{0}}$ is concave with $V_{m}^{b_{0}}\left(b_{0}\right)=1$. Because $V_{m}^{b_{0}}(0) \leq \frac{1}{\alpha}$, it is easy to check that $V^{b_{0}}$ satisfies the assumptions of Proposition 3.3. As a consequence, the optimal strategy is to distribute all the surplus above $b_{0}$ as dividends, to do not hedge and to default the first time the cash reserves hit zero.

Remark 3.2 We have an explicit bound $\bar{p}$ on the hedging cost $p$, that is $\bar{p}=\frac{2 \sigma_{h}^{2} \mu}{\sigma_{h}^{2}+\sigma^{2}}$ above which it is optimal to never hedge. For $p \geq \bar{p}$, the decision to issue equity depends only on the level of issuance costs $\alpha$. The threshold $\bar{p}$ increases with the profitability $\mu$ meaning that the most profitable firm can afford a higher hedging cost but decreases with the idiosyncratic risk $\sigma$. Finally, $\bar{p}$ increases with the market risk $\sigma_{h}$.

Figure 3.1 gives an illustration of the optimal equity issuance policy. In the yellow area, the firm goes bankrupt when the cash reserves hit zero regardless of the hedging policy. Namely, when $\alpha$ is smaller than 0.15 , the issuance cost are so high that an optimal hedging policy does not prevent the firm to default. In the brown area, the optimal hedging offsets the issuance cost and for $\alpha$ between 0.15 and 0.26 there is a level $p(\alpha)$ of hedging cost below which it is optimal to issue equity to avoid bankruptcy. In the dark area, the equity issuance is always optimal regardless of the hedging policy. To conclude, Figure 3.1 highlights the impact of an optimal hedging policy on the permanence of firms when external funding is costly. 


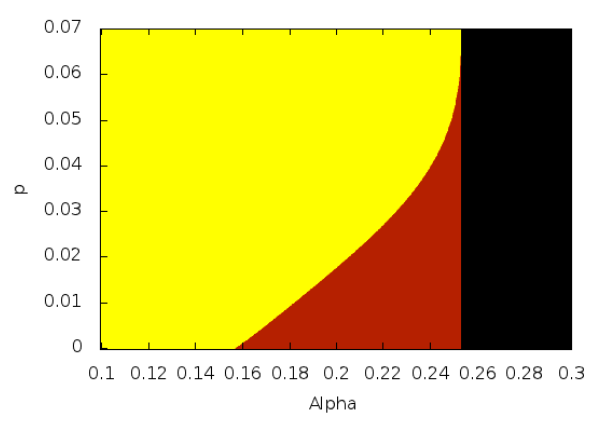

(a) Emission and Hedging

Figure 1: Issuance or bankruptcy zone depending on $p$ and $\alpha$.

Assume now that the investment has been undertaken $u=1$. Regardless of the level of self-financing $a$, the cash holdings will evolve independently of $a$ after the investment decision time $T$ as

$$
\begin{aligned}
d M_{t} & =(r-\delta) M_{t} d t+d X_{t}-2 \psi_{t}\left(\sigma_{h} B_{t}^{h}+p d t\right) \\
& -d Z_{t}+\alpha d R_{t}
\end{aligned}
$$

Proceeding analogously as in Proposition 3.3, we can prove that the shareholders value function $F$ after the investment has been made is the concave solution to

$$
\max _{\psi \in(0,1]} \tilde{L}(\psi) F=\max _{\psi \in(0,1]}\left\{\left(2\left(\sigma^{2}+(1-\psi)^{2} \sigma_{h}^{2}\right)\right) F_{m m}+[(r-\delta) m+2 \mu-2 \psi p] F_{m}-r F\right\}=0
$$

under the boundary conditions

$$
F_{m}\left(b^{*}\right)=1, F_{m m}\left(b^{*}\right)=0 \text { and } \max \left(-F(0), F_{m}(0)-\frac{1}{\alpha}\right)=0 .
$$

In order to simplify the exposition, we will assume that $\alpha$ is enough closed to one in order to have $F_{m}(0)-\frac{1}{\alpha}=0$ and thus $F(0)>0$. Again, we can decompose the operator $\tilde{L}(\psi)$ as follows

$$
\tilde{L}(\psi) F(m)=L(0) F(m)-2 \psi(2-\psi) \sigma_{h}^{2} F_{m m}(m)-2 \psi p F_{m}(m) .
$$

In that case, the first order condition gives

$$
\psi^{*}(m)=1+\frac{p}{2 \sigma_{h}^{2}} \frac{F_{m}}{F_{m m}}(m) .
$$

Again, two cases have to be considered

1. $2 \sigma_{h}^{2} F_{m m}(m)+p F_{m}(m) \geq 0$ where it is optimal to not hedge.

2. $2 \sigma_{h}^{2} F_{m m}(m)+p F_{m}(m) \leq 0$ where it is optimal to hedge according to the policy $\psi^{*}$. 
The value function $F$ can be split in the same manner than in the scenario $u=0$ and we have to solve first on $\left(\tilde{b}, b^{*}\right)$

$$
2\left(\sigma^{2}+\sigma_{h}^{2}\right) F_{m m}+[(r-\delta) m+2 \mu] F_{m}-r F=0
$$

under the boundary conditions

$$
F_{m}\left(b^{*}\right)=1, F_{m m}\left(b^{*}\right)=0,
$$

and secondly, solve on $(0, \tilde{b})$ the non-linear ordinary differential equation

$$
2 \sigma^{2} F_{m m}+[(r-\delta) m+2 \mu-2 p] F_{m}-r F-\frac{p^{2}}{2 \sigma_{h}^{2}} \frac{\left(F_{m}\right)^{2}}{F_{m m}}(m)=0
$$

with the boundary condition $F_{m}(0)=\frac{1}{\alpha}$.

Note that under the assumption $\alpha \leq \beta$, there exists a level of cash $m_{\beta}$ such that $F_{m}\left(m_{\beta}\right)=$ $\frac{1}{\beta}$. The value function at time $T$ is thus $V\left(1, M_{T}\right)$ where

$$
V(1, m)=\max \left(W^{0}(m), \max _{0 \leq a \leq I \wedge m}\left(F(m-a)-\frac{I-a}{\beta}\right)\right) .
$$

For convenience, we denote the shareholder value function if the decision to invest has been made by $W^{1}(m)=\max _{0 \leq a \leq I \wedge m}\left(F(m-a)-\frac{I-a}{\beta}\right)$.

We are now in a position to determine the optimal level of self-financing $a^{*}$ as a function of the cash reserves.

Proposition 3.5 The optimal level of self-financing is given by

$$
a^{*}(m)=\left\{\begin{array}{cc}
0 & \text { if } m \leq m_{\beta} \\
m-m_{\beta} & \text { if } m_{\beta} \leq m \leq m_{\beta}+I \\
I & \text { if } m \geq m_{\beta}+I
\end{array}\right.
$$

Therefore, $W^{1}(m)$ can be decomposed as follows

$$
W^{1}(m)=\left\{\begin{array}{cc}
F(m)-\frac{I}{\beta} & \text { if } m \leq m_{\beta} \\
F\left(m_{\beta}\right)-\frac{I}{\beta}+\frac{m-m_{\beta}}{\beta} & \text { if } m_{\beta} \leq m \leq m_{\beta}+I \\
F(m-I) & \text { if } m \geq m_{\beta}+I
\end{array}\right.
$$

Note that $W^{1}$ is a continuously differentiable function.

Proof: The first order condition gives

$$
\frac{1}{\beta}-F_{m}(m-a)= \begin{cases}\geq 0 & \text { if } m-a \geq m_{\beta} \\ =0 & \text { if } m-a=m_{\beta} \\ \leq 0 & \text { if } m-a \leq m_{\beta}\end{cases}
$$

from which we deduce the optimal level of self-financing.

As soon as the investment opportunity is available, the decision maker has to choose if he undertakes the growth opportunity by comparing $W^{0}$ and $W^{1}$. Therefore, the value function after the arrival date $T$ is $V\left(1, M_{T}\right)$ where

$$
V(1, m)=\max \left(W^{0}(m), W^{1}(m)\right)
$$




\subsection{Before the arrival date of the investment opportunity}

Applying the dynamic programming principle, we observe that $V(0, m)$ can be written

$$
V(0, m)=\sup _{\pi \in \mathcal{A}}\left\{\mathbb{E}\left(\int_{0}^{T \wedge \tau_{B}} e^{-r s}\left(d Z_{s}-d R_{s}\right)\right)+\mathbb{E}\left(e^{-r T} V\left(1, M_{T}^{0}\right) \mathbb{1}_{T \leq \tau_{B}}\right)\right\}
$$

where

$$
d M_{t}^{0}=\left((r-\delta) M_{t}^{0}+\mu\right) d t+\left(\sigma d B_{t}+\sigma_{h} d B_{t}^{h}\right)-\psi\left(\sigma_{h} d B_{t}^{h}+p d t\right)-d Z_{t}+\alpha d R_{t}
$$

But on the other hand,

$$
W^{0}(m)=\sup _{\pi \in \mathcal{A}}\left\{\mathbb{E}\left(\int_{0}^{T \wedge \tau_{B}} e^{-r s}\left(d Z_{s}-d R_{s}\right)\right)+\mathbb{E}\left(e^{-r T} W^{0}\left(M_{T}^{0}\right) \mathbb{1}_{T \leq \tau_{B}}\right)\right\} .
$$

Because $V(1, m) \geq W^{0}(m)$, we obtain that $V(0, m) \geq W^{0}(m)$ which means that ex-ante the growth opportunity is worthwhile.

Using the arguments preceeding Proposition 3.3, we expect that the ex-ante shareholders value function $V(0, m)$ satisfies

$$
\max \left(\max _{\psi \in(0,1]} L(\psi) V(0, m)+\lambda(V(1, m)-V(0, m)), 1-V_{m}(0, m), V_{m}(0, m)-\frac{1}{\alpha}\right)=0
$$

under the boundary conditions

$$
V_{m}(0,0)=\frac{1}{\alpha}
$$

Note that $V\left(0, m\right.$ is positive because $V(0, m) \geq W^{0}(m)>0$ for $\alpha$ satisfying the assumption of Proposition 3.3. The following proposition establishes the result.

Proposition 3.6 Assume there exists a function $W(n, m)$ and a threshold $m_{0}^{*}$ such that

1. $W(0, m)$ is a twice differentiable solution on $(0, \infty)$ of

$$
\max _{\psi \in(0,1]} L(\psi) W(0, m)+\lambda\left(V(1, m)-W(0, m)=0 \text { and } W_{m}(0, m) \geq 1, W_{m}(0, m) \leq \frac{1}{\alpha} \text { for } m \leq m_{0}^{*}\right.
$$

$$
W_{m}(0, m)=1 \text { for } m \geq m_{0}^{*}
$$

together with the initial condition:

$$
W(0,0) \geq 0 \text { and } W_{m}(0,0)=\frac{1}{\alpha},
$$

2. $W(1, m)=V(1, m)$.

then $W(0, m)=V(0, m)$. 
Proof: The proof relies on the same idea as in the one of Proposition 3.3. The main difference comes from the fact that we do not assume that $W(0,$.$) is concave. Fix a policy$ $\pi=\left(\psi_{t}, Z_{t}, R_{t}, a, \tau_{B}\right) \in \mathcal{A}$ and write as usual the processes $Z_{t}=Z_{t}^{c}+Z_{t}^{d}$ and $R_{t}=R_{t}^{c}+R_{t}^{d}$.

Let:

$$
\begin{aligned}
d M_{t} & =(r-\delta) M_{t} d t+\mu d t+\sigma d B_{t}+\sigma_{h} d B_{t}^{h}-\psi_{t}\left(\sigma_{h} d B_{t}^{h}+p d t\right) \\
& -d Z_{t}+\alpha d R_{t} \\
M_{0} & =m
\end{aligned}
$$

be the dynamics of cash reserves under the policy $\pi$ and $N_{t}=\mathbb{1}_{\{t \geq T\}}$. Using again the generalized Itô formula, we can write for all $t \geq 0$,

$$
\begin{aligned}
e^{-r\left(t \wedge T \wedge \tau_{B}\right)} W\left(N_{\left(t \wedge T \wedge \tau_{B}\right)}, M_{\left(t \wedge T \wedge \tau_{B}\right)}\right) & =W(0, m)+\int_{0}^{\left(t \wedge T \wedge \tau_{B}\right)} e^{-r s} L\left(\psi_{s}\right) W\left(N_{s}, M_{s}\right) d s \\
& +\int_{0}^{\left(t \wedge T \wedge \tau_{B}\right)} e^{-r s} W_{m}\left(N_{s}, M_{s}\right)\left(\sigma d B_{t}+\sigma_{h} d B_{t}^{h}\right) \\
& -\int_{0}^{\left(t \wedge T \wedge \tau_{B}\right)} e^{-r s} W_{m}\left(N_{s}, M_{s}\right) d Z_{s}^{c} \\
& +\int_{0}^{\left(t \wedge T \wedge \tau_{B}\right)} e^{-r s} W_{m}\left(N_{s}, M_{s}\right) \alpha d R_{s}^{c} \\
& +\sum_{s \leq\left(t \wedge T \wedge \tau_{B}\right)} e^{-r s}\left(W\left(N_{s}, M_{s}\right)-W\left(N_{s}, M_{s-}\right)\right) \\
& +\int_{0}^{\left(t \wedge T \wedge \tau_{B}\right)} e^{-r s}\left(W\left(N_{s}, M_{s}\right)-W\left(N_{s-}, M_{s}\right)\right) d N_{s},
\end{aligned}
$$

Introducing the martingale

$$
H_{t}=N_{t}-\int_{0}^{t \wedge T} \lambda d s
$$

We obtain

$$
\begin{aligned}
e^{-r\left(t \wedge T \wedge \tau_{B}\right)} W\left(N_{\left(t \wedge T \wedge \tau_{B}\right)}, M_{\left(t \wedge T \wedge \tau_{B}\right)}\right) & =W(0, m) \\
& +\int_{0}^{\left(t \wedge T \wedge \tau_{B}\right)} e^{-r s}\left(L\left(\psi_{s}\right) W\left(N_{s}, M_{s}\right)\right. \\
& +\lambda\left(W\left(1, M_{s}\right)-W\left(0, M_{s}\right)\right) d s \\
& +\int_{0}^{\left(t \wedge T \wedge \tau_{B}\right)} e^{-r s} W_{m}\left(N_{s}, M_{s}\right)\left(\sigma d B_{t}+\sigma_{h} d B_{t}^{h}\right) \\
& -\int_{0}^{\left(t \wedge T \wedge \tau_{B}\right)} e^{-r s} W_{m}\left(N_{s}, M_{s}\right) d Z_{s}^{c} \\
& +\int_{0}^{\left(t \wedge T \wedge \tau_{B}\right)} e^{-r s} W_{m}\left(N_{s}, M_{s}\right) \alpha d R_{s}^{c} \\
& +\sum_{s \leq\left(t \wedge T \wedge \tau_{B}\right)} e^{-r s}\left(W\left(N_{s}, M_{s}\right)-W\left(N_{s}, M_{s-}\right)\right) \\
& +\int_{0}^{\left(t \wedge T \wedge \tau_{B}\right)} e^{-r s}\left(W\left(N_{s}, M_{s}\right)-W\left(N_{s-}, M_{s}\right)\right) d H_{s},
\end{aligned}
$$


Using assumptions on $W(0, m)$, the first integral is non positive and because $W(0, m)$ has a bounded first derivative, the two stochastic integrals are martingales. Finally, the function $W(1, m)-W(0, m)$ is continuous on $[0, \infty)$ with $\lim _{m \rightarrow \infty} W(1, m)-W(0, m)=C$ where $C$ is a constant. Thus $W(1, m)-W(0, m)$ is bounded and the last term is also a martingale. Taking expectations, we get

$$
\begin{aligned}
W(0, m) & \geq E\left(e^{-r\left(t \wedge T \wedge \tau_{B}\right)} W\left(N_{t \wedge T \wedge \tau_{B}}, M_{t \wedge T \wedge \tau_{B}}\right)\right)+E\left(\int_{0}^{\left(t \wedge T \wedge \tau_{B}\right)} e^{-r s} W_{m}\left(N_{s}, M_{s}\right) d Z_{s}^{c}\right) \\
& -E\left(\int_{0}^{\left(t \wedge T \wedge \tau_{B}\right)} e^{-r s} W_{m}\left(N_{s}, M_{s}\right) \alpha d R_{s}^{c}\right) \\
& +E\left(\sum_{s \leq\left(t \wedge T \wedge \tau_{B}\right)} e^{-r s}\left(W\left(N_{s}, M_{s-}\right)-W\left(N_{s}, M_{s}\right)\right)\right)
\end{aligned}
$$

We assume without loss of generality that the processes $Z$ and $R$ have no common jumps and write

$$
\begin{aligned}
W\left(N_{s}, M_{s-}\right)-W\left(N_{s}, M_{s}\right) & =\left(W\left(N_{s}, M_{s-}\right)-W\left(N_{s}, M_{s}\right)\right)\left(\mathbb{1}_{\Delta M_{s}=-\Delta Z_{s}}+\mathbb{1}_{\Delta M_{s}=\alpha \Delta R_{s}}\right) \\
& =\left(\int_{M_{s}}^{M_{s-}} W_{m}\left(N_{s}, u\right) d u\right) \mathbb{1}_{\Delta M_{s}=-\Delta Z_{s}} \\
& +\left(\int_{M_{s}}^{M_{s-}} W_{m}\left(N_{s}, u\right) d u\right) \mathbb{1}_{\Delta M_{s}=\alpha \Delta R_{s}} \\
& \geq-\Delta M_{s} \mathbb{1}_{\Delta M_{s}=-\Delta Z_{s}}+\frac{1}{\alpha} \Delta M_{s} \mathbb{1}_{\Delta M_{s}=\alpha \Delta R_{s}} \\
& =\Delta Z_{s}-\Delta R_{s} .
\end{aligned}
$$

Therefore,

$$
W(0, m) \geq \mathbb{E}\left(\int_{0}^{t \wedge T \wedge \tau_{B}} e^{-r s}\left(d Z_{s}-d R_{s}\right)\right)+\mathbb{E}\left(e^{-r\left(t \wedge T \wedge \tau_{B}\right)} W\left(N_{t \wedge T \wedge \tau_{B}}, M_{t \wedge T \wedge \tau_{B}}\right)\right)
$$

Now,

$$
\begin{aligned}
\mathbb{E}\left(e^{-r\left(t \wedge T \wedge \tau_{B}\right)} W\left(N_{t \wedge T \wedge \tau_{B}}, M_{t \wedge T \wedge \tau_{B}}\right)\right) & =\mathbb{E}\left(e^{-r t} W\left(N_{t}, M_{t}\right) \mathbb{1}_{\left\{t \leq \min \left(T, \tau_{B}\right)\right\}}\right) \\
& +\mathbb{E}\left(e^{-r \tau_{B}} W\left(N_{\tau_{B}}, M_{\tau_{B}}\right) \mathbb{1}_{\left\{\tau_{B} \leq \min (t, T)\right\}}\right) \\
& +\mathbb{E}\left(e^{-r T} W\left(N_{T}, M_{T}\right) \mathbb{1}_{\left\{T \leq \min \left(t, \tau_{B}\right)\right\}}\right)
\end{aligned}
$$

Because, $\left(N_{\tau_{B}}, M_{\tau_{B}}\right)=(0,0)$ on the set $\left\{\tau_{B} \leq T \wedge t\right\}$ and because $W(0,0)$ is positive, the second term of the right-hand-side is positive. Analogously, because $W(0, m)$ has bounded first derivative and $\lim _{t \rightarrow \infty} E\left(e^{-r t} M_{t}^{\pi}\right)=0$ for $\pi \in \mathcal{A}$, the first term vanishes when $t$ tends to $\infty$. Finally, it is obvious that $e^{-r T} V\left(1, M_{T}\right)$ is integrable for $\pi \in \mathcal{A}$ and by the dominated bounded convergence theorem, we get

$$
\begin{aligned}
W(0, m) & \geq \mathbb{E}\left(\int_{0}^{T \wedge \tau_{B}} e^{-r s}\left(d Z_{s}-d R_{s}\right)\right)+\mathbb{E}\left(e^{-r T} V\left(1, M_{T}\right) \mathbb{1}_{T \leq \tau_{B}}\right) \\
& =V(0, m) .
\end{aligned}
$$


The other inequality can be obtained with the same arguments as in proposition 3.3.

\section{Costless hedging}

In this section, we assume that the hedging policy is costless, that is $p=0$. In that case, the cash reserves evolves as

$$
d M_{t}=(r-\delta) M_{t} d t+d X_{t}-d Z_{t}+\alpha d R_{t}-\mathbb{1}_{\{u=1\}} a d N_{t}
$$

where

$$
d X_{t}=K_{t}\left(\mu d t+\sigma d B_{t}\right)
$$

Assume that we are at time $T$ with $V_{T}=m$, the manager acting in the best interest of the shareholders has to decide if he undertakes the growth opportunity $u=1$ or if he maintains the activity in place $u=0$. If he decides to invest, he has also to decide the amount $a$ of self-financing.

The manager will decide to maintain the activity in place if and only if the maximum value in (3.21) is $W^{0}$. We study now a set of conditions to ensure the optimality of the investment in the growth opportunity.

For large $m$, more precisely for $m \geq \max \left(b_{0}^{*}, b^{*}+I\right)$ we observe that

$$
W^{0}(m)=m+\frac{\mu}{r}-\frac{\delta}{r} b_{0}^{*}
$$

and

$$
W^{1}(m)=F(m-I)=m-I+\frac{2 \mu}{r}-\frac{\delta}{r} b^{*} .
$$

A necessary condition to make the opportunity worthless $\left(W^{0} \geq W^{1}\right)$ is

$$
b^{*}-b_{0}^{*} \geq \frac{\mu-r I}{\delta}
$$

Note that this condition is equivalent to the condition given in [7], Proposition 2.2 and [12], Proposition 3. Next Proposition shows that condition (4.26) is also sufficient to make the opportunity worthless.

Proposition 4.7 The growth opportunity is worthless $\left(W^{0}(m) \geq W^{1}(m)\right)$ if and only if Condition (4.26) is satisfied.

The proof of Proposition 4.7 relies on the following lemma.

Lemma 4.1 The function $W^{0}-F$ is a decreasing function.

Proof: Let $k=W^{0}-F . \quad k$ is infinitely differentiable on $\left(0, b_{0}^{*}\right)$ under condition (4.26). Let us define the differential operator $\tilde{L}$ by

$$
\tilde{L} f=2 \sigma^{2} f_{m m}+[(r-\delta) m+2 \mu] f_{m}-\delta f
$$


Note that $F_{m}$ satisfies $\tilde{L} F_{m}=0$ on $\left(0, b_{0}^{*}\right)$. On the other hand,

$$
\tilde{L} W_{m}^{0}=3 \delta W_{m}^{0}-(3(r-\delta) m+2 \mu) W_{m m}^{0} .
$$

Therefore, $\tilde{L} k_{m} \geq 0$ on $\left(0, b_{0}^{*}\right)$. Because, $k_{m}(0)=0$ and $k_{m}\left(b_{0}^{*}\right)<0$ under condition (4.26), the maximum principle gives that $k_{m}$ is non positive on $\left(0, b_{0}^{*}\right)$ and thus on $\mathbb{R}_{+}$.

Proof of Proposition 4.7: It is clear that $W^{1}$ is always bounded by $F(x)-I$ which corresponds to the value function after $T$ for $\beta=1$. According to Lemma 4.1, the function $W^{0}-(F-I)$ is decreasing. Because, $W^{0}\left(b_{0}^{*}\right) \geq F\left(b_{0}^{*}\right)-I$ under condition (4.26), we deduce that $W^{0} \geq F-I$ everywhere and thus $W^{0} \geq W^{1}$.

Under condition (4.26) and assumption (2.1), we observe that the level of dividend distribution $b^{*}$ corresponding to the decision to invest is larger than the level of dividend distribution $b_{0}^{*}$ corresponding to the decision to not grasp the growth opportunity. Next Proposition proves that condition (4.26) is not necessary.

Proposition 4.8 We have $b^{*} \geq b_{0}^{*}$.

Proof: Assume the contrary and still denote $k=W^{0}-F$. Because $b^{*}<b_{0}^{*}$ is assumed, we have $k_{m}(0)=0, k_{m}\left(b^{*}\right)>0$ and $k_{m m}\left(b^{*}\right)<0$. Therefore, there is some $y$ such that $k_{m m}(y)=0$ with $k_{m}(y)>0$ and $k_{m m m}(y) \leq 0$ and thus $\tilde{L} k_{m}(y)<0$. But, we still have

$$
\tilde{L}\left(k_{m}\right)=3 \delta W_{m}^{0}-(3(r-\delta) m+2 \mu) W_{m m}^{0} \geq 0
$$

which yields to a contradiction.

$\diamond$

The last proposition deserves some comments about the impact of both profitability and volatility on the dividend distribution. For convenience, we use the notation $b_{0}^{*}=b(\mu, \sigma)$ and $b^{*}=b(2 \mu, 2 \sigma)$. According to [6], we know that the optimal threshold $b$ is an increasing function of $\sigma$ and a decreasing function of $\mu$. Proposition 4.8 shows that the volatility effect dominates the profitability effect when assuming constant return to scale since $b(\mu, \sigma) \leq$ $b(2 \mu, 2 \sigma)$.

Next Proposition shows that the growth opportunity is worthwhile if and only if the cash reserves are important enough which give a new insight on the role of cash reserve.

Proposition 4.9 The function $W^{0}-W^{1}$ is decreasing.

Proof: Let define $k=W^{0}-W^{1}$. The idea is again to apply the maximum principle but we have to circumvent the fact that $W^{1}$ is not a twice differentiable function. The derivative of $k$ is a continuous function on $\left(0, b_{0}^{*}\right)$ that is $C^{2}$ almost everywhere except for $m=m_{\beta}$ and $m=m_{\beta}+I$. A direct computation shows that $\tilde{L}\left[k_{m}\right]$ is non negative at any point $m$ where $k_{m}$ is twice differentiable. Indeed,

- for $0<m<m_{\beta}, W^{1}(m)=F(m)-I$ thus $\tilde{L}\left[\left(W^{1}\right)_{m}\right](m)=0$. Therefore,

$$
\tilde{L}\left[k_{m}\right]=-[3(r-\delta) m+2 \mu] W_{m m}^{0}+3 \delta W_{m}^{0} \geq 0 .
$$


- for $m_{\beta}<m<m_{\beta}+I,\left(W^{1}\right)_{m}=\frac{1}{\beta}$ and thus

$$
\tilde{L}\left[k_{m}\right]=-[3(r-\delta) m+2 \mu] W_{m m}^{0}+3 \delta W_{m}^{0}+\frac{\delta}{\beta} \geq 0
$$

- for $m>m_{\beta}+I, \tilde{L}\left[\left(W^{1}\right)_{m}\right](m)=(r-\delta) I F_{m m}(m-I)$ and therefore

$$
\tilde{L}\left[k_{m}\right]=-[3(r-\delta) m+2 \mu] W_{m m}^{0}+3 \delta W_{m}^{0}-(r-\delta) I F_{m m}(m-I) \geq 0 .
$$

We have $k_{m}(0)=0, k_{m}\left(m_{\beta}\right)=W_{m}^{0}\left(m_{\beta}\right)-\frac{1}{\beta} \leq 0$ according to Lemma 4.1. Let introduce $\phi(x)=W_{m}^{0}\left(m_{\beta}+x\right)-\frac{1}{\beta}$. Because $W^{0}$ concave, we have that $\phi$ is a decreasing function with $\phi(0) \leq 0$ thus $\phi(I) \leq 0$ which implies that $k_{m}\left(m_{\beta}+I\right) \leq 0$. Besides $k_{m}\left(b^{*}+I\right) \leq 0$. We end the demonstration by applying the maximum principle on $\left[0, m_{\beta}\right],\left[m_{\beta}, m_{\beta}+I\right]$, and $\left[m_{\beta}, m_{\beta}+I\right]$ separately which gives that $k_{m}$ is negative one each interval.

According to Proposition 4.9, there is a threshold $\tilde{m}$ defined by $W^{0}(\tilde{m})=W^{1}(\tilde{m})$ above which the growth opportunity is worthwhile when Condition (4.26) is not fulfilled. In the presence of investment opportunity, $\tilde{m}$ can be viewed as a target level for management above which the irreversible opportunity to double the size of asset is worthwhile.

Proposition 4.9 has also a nice consequence of the behavior of $V(0, m)$ for large value of cash reserves.

Proposition 4.10 We have

$$
\lim _{m \rightarrow+\infty} \frac{V(0, m)}{m}=1
$$

Proof: For large $m$, we have $W^{1}(m)=W^{0}(m)+C$ where $C$ is an explicit constant. Using Proposition 4.9, we deduce that $W^{1}(m) \leq W^{0}(m)+C$ for all $m>0$. Using the dynamic programming principle (3.22), we have that

$$
V(0, m) \leq W^{0}(m)+C
$$

Using $V(0, m) \geq m$, we get the result because

$$
\lim _{m \rightarrow+\infty} \frac{W^{0}(m)}{m}=1
$$

Next Proposition is a first step in the explicit construction of the value function before the arrival date of the investment opportunity. Let us define

$$
L_{\lambda} f=\frac{\sigma^{2}}{2} f_{m m}+((r-\delta) x+\mu) f_{m}-(r+\lambda) f .
$$

Proposition 4.11 There exists a twice differentiable function $\hat{V}$ and a threshold $b_{0, \lambda}^{*}$ such that $L_{\lambda} \hat{V}+\lambda V(1, m)=0,(\hat{V})_{m}\left(b_{0, \lambda}^{*}\right)=1,(\hat{V})_{m m}\left(b_{0, \lambda}^{*}\right)=0$ and $(\hat{V})_{m}(0)=\frac{1}{\alpha}$. 
Proof: If $V(1, m)=W^{0}$ then it is obvious that $W^{0}$ and $b_{0}^{*}$ satisfies Proposition 4.11. We thus assume that $V\left(1, b^{*}+I\right)=W^{1}\left(b^{*}+I\right)$. We fix $b>b^{*}+I$ and define $W^{b}$ the solution of

$$
L_{\lambda} W^{b}+\lambda V(1, m)=0 \quad W_{m}^{b}(b)=1 W_{m m}^{b}(b)=0 .
$$

We first prove that $W^{b}$ is concave on $\left(b^{*}+I, b\right)$.

Because $W_{m m m}^{b}(b)=\frac{2 \delta}{\sigma^{2}}$, the function $W^{b}$ is concave in a left neighborhood of $b$. Assume that there is some $y \in\left(b^{*}+I, b\right)$ such that $W_{m m}^{b}(y)=0$ and $W_{m m}^{b}(x)<0$ for $\left.x \in\right] y, b[$. We have $W_{m}^{b}(y) \geq 1$ and thus

$$
((r-\delta) y+\mu)-(r+\lambda) W^{b}(y)+\lambda V(1, y) \leq 0
$$

On the other hand,

$$
((r-\delta) b+\mu)-(r+\lambda) W^{b}(b)+\lambda V(1, b)=0 .
$$

Substracting the last two inequalities, we get because $V(1, m)$ is linear on $\left(b^{*}+I, b\right)$,

$$
\frac{W^{b}(b)-W^{b}(y)}{b-y} \leq 1-\frac{\delta}{r+\lambda}
$$

which yields to a contradiction. Therefore, $W^{b}$ is concave on $\left(b^{*}+I, b\right)$.

Now, let us define $k^{l}=W^{b+l}-F(.-I-l)$ for $l>0$ where $F$ has been extended on $[-I-l, 0] . F$ is a still a concave function on $\left[-I-l, b^{*}\right]$ and $F_{m}(-I-l)$ goes to infinity as $l$ goes to infinity. We have

$L_{\lambda} k^{l}=-\left(\frac{3}{4}(r-\delta) m+\frac{(r-\delta)(I+l)}{4}+\frac{\mu}{2}\right) F_{m}(m-I-l)+\frac{3}{4} r F(m-I-l)-\lambda\left(V^{1}(1, m)-F(m-I-l)\right)$

and

$$
\begin{aligned}
\left(L_{\lambda}+(r-\delta)\right) k_{m}^{l} & =-\left(\frac{3}{4}(r-\delta) m+\frac{(r-\delta)(I+l)}{4}+\frac{\mu}{2}\right) F_{m m}(m-I-l)+\frac{3}{4} \delta F(m-I-l) \\
& -\lambda\left(V_{m}^{1}(1, .)-F_{m}(m-I-l)\right)
\end{aligned}
$$

A straightforward computation yields to $V_{m}^{1}(1, m)-F_{m}(m-I-l)=F_{m}(m-I)-F_{m}(m-$ $I-l)<0$ for $m \geq m_{\beta}+I$, $V_{m}^{1}(1, m)-F_{m}(m-I)=1 / m_{\beta}-F_{m}(m-I-l) \leq 0$ for $m \in\left(m_{\beta}, m_{\beta}+I\right)$ and $V_{m}^{1}(1, m)-F_{m}(m-I-l)=F_{m}(m)-F_{m}(m-I-l)<0$ for $m \leq m_{\beta}$. Therefore, $\left(L_{\lambda}+(r-\delta)\right) k_{m}^{l} \geq 0$. Because $W^{b+l}$ is strictly concave on $\left(b^{*}+I, b+l\right)$, $k_{m m}^{l}\left(b^{*}+I+l\right)<0$ and $k_{m}^{l}\left(b^{*}+I+l\right)>0$. Now, two cases have to be considered.

- First case : $V(1, m)=W^{1}(m)$, the maximum principle on $\left[0, b^{*}+I\right]$ implies that $k_{m}^{l}(0)>0$ which is equivalent to $W_{m}^{b}(0) \geq \frac{1}{\alpha}$.

- Second case: according to the maximum principle on $\left[\tilde{m}, b^{*}+I\right], k_{m}^{l}(\tilde{m})>0$ so $W_{m}^{b+l}(\tilde{m})>F_{m}(\tilde{m}-I-l)$. Because $F_{m}(-I-l)$ goes to infinity as $l$ goes to infinity, 
$F_{m}(\tilde{m}-I-l)>W_{m}^{0}(\tilde{m})$ for $l$ large enough. Introduce $h^{l}=W^{b+l}-W^{0}$. For $l$ large enough, the function $h^{l}$ satisfies

$$
L_{\lambda} h^{l}=0
$$

so

$$
\left(L_{\lambda}+(r-\delta)\right) h_{m}^{l}=0
$$

and

$$
h_{m}^{l}(\tilde{m}) \geq 0
$$

Applying again the maximum principle between 0 and $\tilde{m}$, we obtain that $W_{m}^{b+l}(0) \geq \frac{1}{\alpha}$ for $l$ large enough.

The function $b \rightarrow W_{m}^{b}(0)$ is continuous (Cauchy-Lipschitz Theorem) and because $W_{m}^{0}(0)=$ 1 there is $b_{0, \lambda}^{*}$ such that $W_{m}^{b_{0, \lambda}^{*}}(0)=\frac{1}{\alpha}$.

Note that if the solution $\hat{V}$ of Proposition 4.11 is concave then the shareholders value function is $V(0, m)=\hat{V}(m)$ according to Proposition 3.6. The next two Propositions give sufficient conditions for the concavity of $\hat{V}$.

Proposition 4.12 If $\frac{\delta}{\lambda} \geq \frac{1-\alpha}{\alpha}$ then $\hat{V}$ is concave.

Proof: Assume there is some $x \leq b_{0, \lambda}^{*}$ such that $\hat{V}_{m m}(x)=0$ and $\hat{V}_{m m}<0$ on $] x, b_{0, \lambda}^{*}[$. Because $\hat{V}$ is concave on $\left(x, b_{0, \lambda}^{*}\right)$, we must have $\hat{V}_{m}(x) \geq 1$ and $\hat{V}_{m m m}(x) \leq 0$.

First, suppose that $x \neq \tilde{m}$. Therefore, $V(1, m)$ is differentiable at $x$ and thus

$$
\frac{\sigma^{2}}{2} \hat{V}_{m m m}(x)-(\delta+\lambda) \hat{V}_{m}(x)+\lambda V_{m}(1, x)=0 .
$$

This implies

$$
V_{x}(1, x) \geq 1+\frac{\delta}{\lambda}
$$

Now, suppose that $x=\tilde{m}$. We introduce the function

$$
\phi(m)=\frac{\lambda V(1, m)+[(r-\delta) m+\mu] \hat{V}_{m}(m)}{r+\lambda} .
$$

By assumption, $\phi$ is differentiable on $\left(x, b_{0, \lambda}^{*}\right)$ and we have $\hat{V}\left(b_{0, \lambda}^{*}\right)=\phi\left(b_{0, \lambda}^{*}\right)$ and $\hat{V}(x)=$ $\phi(x)$. By Rolle's theorem, there exists $y \in\left(x, b_{0, \lambda}^{*}\right)$ such that $\hat{V}_{m}(y)=\phi_{m}(y)$ yielding as in the first case (because $\hat{V}$ is concave on $y \in\left(x, b_{0, \lambda}^{*}\right)$ )

$$
V_{m}(1, x) \geq 1+\frac{\delta}{\lambda}
$$

Using the hypothesis $\frac{\delta}{\lambda} \geq \frac{1-\alpha}{\alpha}$, we get $V_{m}(1, x) \geq \frac{1}{\alpha}$ which yields to a contradiction and ends the proof.

Proposition 4.13 If the set of parameters are such that $V(1, m)=W^{1}(m)$ then $\hat{V}$ is concave and thus $V(0, m)=\hat{V}(m)$. 
Proof: Assume that there is some $x_{0}<b_{0, \lambda}^{*}$ such that $\hat{V}_{m m}\left(x_{0}\right)=0$ and $\hat{V}_{m m}(x) \leq 0$ for $x \in\left(x_{0}, b_{0, \lambda}^{*}\right)$. First, we will prove that $\hat{V}_{m}\left(x_{0}\right)<\frac{1}{\alpha}$. Differentiating the o.d.e, we get

$$
\frac{\sigma^{2}}{2} \hat{V}_{m m m}\left(x_{0}\right)-(\delta+\lambda) \hat{V}_{m}\left(x_{0}\right)+\lambda W_{m}^{1}\left(x_{0}\right)=0 .
$$

Because $\hat{V}_{m m m}\left(x_{0}\right) \leq 0$, we must have

$$
\hat{V}_{m}\left(x_{0}\right) \leq \frac{\lambda}{\lambda+\delta} W_{m}^{1}\left(x_{0}\right) \leq \frac{\lambda}{\lambda+\delta} \frac{1}{\alpha}<\frac{1}{\alpha} .
$$

Now, the boundary condition $\hat{V}_{m}(0)=\frac{1}{\alpha}$ implies that $\hat{V}$ cannot be convex on $\left(0, x_{0}\right)$ because $\hat{V}_{m}\left(x_{0}\right)<\frac{1}{\alpha}$. Thus, there is some $y_{0}<x_{0}$ such that $\hat{V}_{m m}\left(y_{0}\right)=0$ and $\hat{V}$ strictly convex on $\left(y_{0}, x_{0}\right)$. As a consequence, $\hat{V}_{m m m}\left(y_{0}\right) \geq 0$. Because $W^{1}$ is concave, the function

$$
k(x)=\frac{\sigma^{2}}{2} \hat{V}_{m m m}(x)+((r-\delta) x+\mu) \hat{V}_{m m}(x)-(\delta+\lambda) \hat{V}_{m}(x)
$$

is increasing and thus $k\left(y_{0}\right) \leq k\left(x_{0}\right)$.

But,

$$
k\left(y_{0}\right)=\frac{\sigma^{2}}{2} \hat{V}_{m m m}\left(y_{0}\right)-(\delta+\lambda) \hat{V}_{m}\left(y_{0}\right) \geq-(\delta+\lambda) \hat{V}_{m}\left(y_{0}\right)
$$

and

$$
k\left(x_{0}\right)=\frac{\sigma^{2}}{2} \hat{V}_{m m m}\left(x_{0}\right)-(\delta+\lambda) \hat{V}_{m}\left(x_{0}\right) \leq-(\delta+\lambda) \hat{V}_{m}\left(x_{0}\right) .
$$

Therefore, $k$ increasing implies $\hat{V}_{m}\left(x_{0}\right) \leq \hat{V}_{m}\left(y_{0}\right)$ which contradicts $\hat{V}$ strictly convex on $\left(y_{0}, x_{0}\right)$. Therefore, $x_{0}$ does not exist and $\hat{V}$ is concave.

We are now in a position to prove the main result of this section. Next Proposition establishes the existence of a solution to the free boundary problem of Proposition 3.6. Let

$$
L_{\lambda}(\psi) f=\frac{\sigma^{2}+(1-\psi)^{2} \sigma_{h}^{2}}{2} f_{m m}+((r-\delta) x+\mu) f_{m}-(r+\lambda) f .
$$

Proposition 4.14 There exists a twice differentiable function $U$ and a threshold $m_{0}^{*}$ such that

$$
\max \left(L_{\lambda}(0), L_{\lambda}(1)\right) U(m)+\lambda V(1, m)=0
$$

and

$$
(U)_{m}\left(m_{0}^{*}\right)=1,(U)_{m m}\left(m_{0}^{*}\right)=0 \text { and }(U)_{m}(0)=\frac{1}{\alpha} .
$$

Moreover, the value function $V(0, m)$ coincides with $U(m)$.

Proof: The proof relies on the study of the following boundary value problem

$$
\max \left(L_{\lambda}(0), L_{\lambda}(1)\right) U^{b}(m)+\lambda V(1, m)=0,\left(U^{b}\right)_{m}(b)=1,\left(U^{b}\right)_{m m}(b)=0 .
$$

First, we prove that $U^{b}$ is concave for $b$ large enough. Let $V^{b}$ the solution of

$$
L_{\lambda}(1) V^{b}(m)+\lambda V(1, m)=0,\left(V^{b}\right)_{m}(b)=1,\left(V^{b}\right)_{m m}(b)=0 .
$$


Note that if the function $V^{b}$ is concave then $U^{b}=V^{b}$. We know that $V^{b}$ is concave in a left neighborhood of $b$. Assume by a way of contradiction that there is some $m_{b}$ such that $V_{m m}^{b}\left(m_{b}\right)=0$. Due to the Proposition 4.11, $m_{b}<b^{*}+I$. Thus $\left(V^{b}\right)_{m}(m) \geq 1$ for all $m \in\left(m_{b}, b\right)$. For $b>b^{*}+I>m \geq m_{b}$,

$$
\begin{aligned}
\frac{\sigma^{2}}{2} V_{m m}^{b}(m) & =\frac{\sigma^{2}}{2}\left(V_{m m}^{b}(m)-V_{m m}^{b}(b)\right) \\
& \leq\left((r+\lambda)\left(V^{b}(m)-V^{b}(b)\right)-(r-\delta)(m-b)+\lambda(V(1, b)-V(1, m))\right) \\
& \leq(\delta+\lambda)(m-b)+\lambda(V(1, b)-V(1, m)) \\
& =(\delta+\lambda)(m-b)+\lambda\left(V(1, b)-V\left(1, b^{*}+I\right)+V\left(1, b^{*}+I\right)-V(1, m)\right) \\
& =(\delta+\lambda)(m-b)+\lambda\left(b-\left(b^{*}+I\right)+V\left(1, b^{*}+I\right)-V(1, m)\right) \\
& <\delta\left(b^{*}+I-b\right)+\lambda\left(m-\left(b^{*}+I\right)\right)\left(1-\frac{1}{\alpha}\right) \\
& <\left(b^{*}+I\right)\left(\delta+\lambda\left(\frac{1}{\alpha}-1\right)\right)-\delta b .
\end{aligned}
$$

Therefore, for $b \geq \frac{\left(b^{*}+I\right)\left(\delta+\lambda\left(\frac{1}{\alpha}-1\right)\right)}{\delta},\left(V^{b}\right)_{m m}\left(m_{b}\right)<0$ which yields to a contradiction.

We have that $U^{b}$ is concave for $b$ large enough and consequently (see Proposition 4.11) $U_{m}^{b}(0) \geq \frac{1}{\alpha}$ for some $b$ large enough.

Now, we prove that there is at most two threshold $x_{0} \leq \tilde{m} \leq x_{1}$ such that $U_{m m}^{b}\left(x_{i}\right)=0$. If if is not the case, $U_{m m}^{b}$ would vanish twice in a region where $V(1, m)$ is concave. Proceeding analogously as in Proposition 4.13, it is straightforward to get a contradiction by working on each region where $V(1, m)$ is concave .

Because the control $\psi$ admits at most two points of discontinuity, the Cauchy-Lipschtiz theorem applies and thus the mapping $b \rightarrow U_{m}^{b}(0)$ is continuous (see Arnold [1]). Consequently, there is some $m_{0}^{*}$ such that $U_{m}^{m_{0}^{*}}(0)=\frac{1}{\alpha}$.

Finally, it remains to show that $V(0, m)=U^{m_{0}^{*}}(m)$ or equivalently that $U_{m}^{m_{0}^{*}}(m) \geq 1$ and $U_{m}^{m_{0}^{*}}(m) \leq \frac{1}{\alpha}$. Let's show the first assertion. If ever, $U^{m_{0}^{*}}$ is concave, there is nothing to prove. Assume thus that there is some $0<x_{0}<\tilde{m}<x_{1}$ such that $U^{m_{0}^{*}}$ is concave on $\left(0, x_{0}\right)$, convex on $\left(x_{0}, x_{1}\right)$ and concave on $\left(x_{1}, m_{0}^{*}\right)$. Note that if we do not have $U_{m}^{m_{0}^{*}} \geq 1$ then by convexity it is equivalent to assume that $U_{m}^{m_{0}^{*}}\left(x_{0}\right)<1$. If it is the case, let us define $k(m)=U_{m}^{m_{0}^{*}}(m)-W_{m}^{0}(m)$. We have $k(0)=0, k\left(x_{0}\right)<0$ and $k_{m m}\left(x_{0}\right) \geq 0$. Thus, there is some $y<x_{0}$ such that $k(y)<0, k_{m}(y)=0$ and $k_{m m}(y) \geq 0$ which yields to a contradiction because $k$ satisfies on $\left(0, x_{0}\right)$

$$
\left.\frac{\sigma^{2}}{2} k_{m m}+(r-\delta) m+\mu\right) k_{m}-(\delta+\lambda) k=0
$$

For the second assertion, the inequality $U_{m}^{m_{0}^{*}} \leq \frac{1}{\alpha}$ can be obtained working on each region where $V(1, m)$ is concave similarly to Proposition 4.13 . 


\section{$5 \quad$ Algorithms and numerical results}

We present in this section all the algorithms developed to compute the value function and the management strategy in terms of hedging, investment, dividend payout and equity issuance before and after the arrival of the investment opportunity. Then numerical results are given and discussed to illustrate the different optimal policies.

\subsection{Algorithms}

After the investment opportunity arrival at date $T$, we have to solve Equations (3.7) and (3.8) that both depend on the optimal hedging $\psi^{*}$. Knowing the boundary level value $b_{0}^{*}$, the concave solution $W$ of proposition 3.4 satisfies the equation $L(0) W=0$ near $b_{0}^{*}$ and can be calculated using a Runge Kutta scheme with step $h$ starting at $b_{0}^{*}$ with initial value $\left(W\left(b_{0}^{*}\right), W_{m}\left(b_{0}^{*}\right)\right)=\left(\frac{(r-\delta) b_{0}^{*}+\mu}{r}, 1\right)$.

The solution of this equation is valid as long as it is optimal to not hedge.

All values $\left((W)\left(b_{0}^{*}-i h\right), W_{m}\left(b_{0}^{*}-i h\right)\right)$ are thus calculated for $i=0$ to $\tilde{i}$ until the solution reaches the domain where hedging is optimal that is when

$$
\psi^{*}\left(b_{0}^{*}-\tilde{i} h\right)=1+\frac{p}{\sigma_{h}^{2}} \frac{\left(W_{m}\right)^{2}}{(W)_{m m}}\left(b_{0}^{*}-\tilde{i} h\right)>0
$$

In order to solve the equation for $m<b_{0}^{*}-\tilde{i} h$, we used an explicit Runge Kutta scheme :

$$
L(0) W(m-h)-p W(m-h)=\frac{p^{2}}{2 \sigma_{h}^{2}} \frac{\left(W_{m}\right)^{2}}{(W)_{m m}}(m)
$$

The function initial value $\left(W(0), W_{m}(0)\right)$ are thus computed .

Algorithm 1 allows us to calculate the value $\left(W^{b}\right)_{m}(0)$ for a given threshold $b$ such that $W^{b}$ satisfies

$$
\forall m \leq b \quad \max _{\psi \in(0,1]} L(\psi) W^{b}=0 \text { and }\left(W^{b}\right)_{m}(b)=1,\left(W^{b}\right)_{m m}(b)=0 .
$$




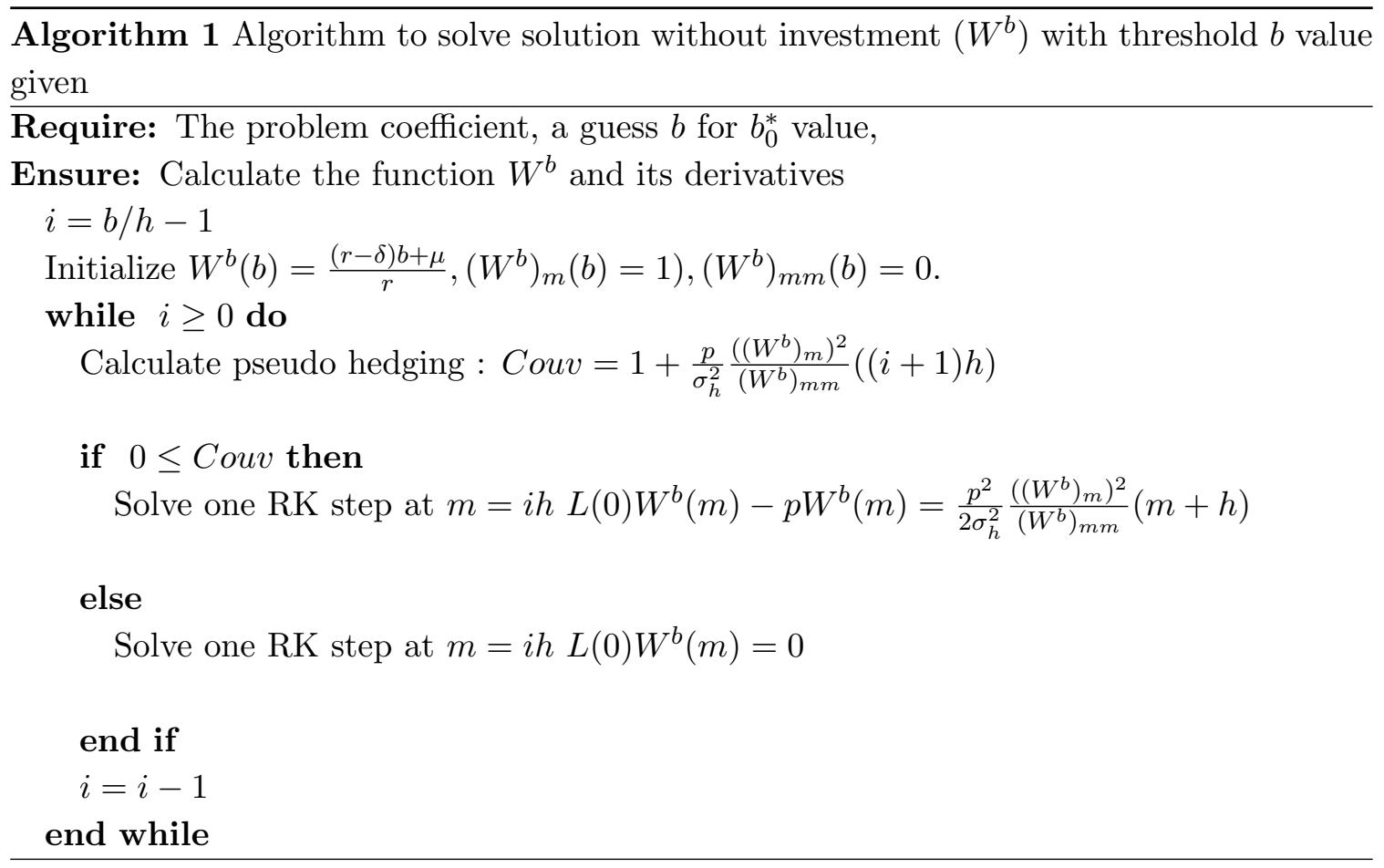

Because the optimal threshold $b_{0}^{*}$ is unknown, we propose a dichotomous scheme based on the empirically observed property that the function $b \longrightarrow\left(W^{b}\right)_{m}(0)$ is increasing (recall that the monotonous property of the initial condition is known to be true for linear operator and seems to be true in the non linear case). Algorithm 2 gives the dichotomous procedure to compute $b_{0}^{*}$. In the case where the computed function $W^{b_{0}^{*}}$ satisfies $W^{b_{0}^{*}}(0)<0$ then a similar dichotomous scheme is used to find $b_{1}^{*}$ such that $W^{b_{1}^{*}}(0)=0$. In that case, the firm defaults when the cash reserves hit 0 .

Same algorithms are used to compute the functions $F$ solution of Equation (3.19) and $W^{1}$ given by Proposition 3.5.

Before the arrival date $T$, a different algorithm has to be used because the function $b \rightarrow\left(V^{b}\right)_{m}(0)$ where $V^{b}$ is solution of

$$
\forall m \leq b \quad \max _{\psi \in(0,1]}(L(\psi)-\lambda) V^{b}(m)=-\lambda V(1, m) \text { and }\left(V^{b}\right)_{m}(b)=1,\left(V^{b}\right)_{m m}(b)=0 .
$$

is very sensitive to the boundary value $b$. Besides we know that $V^{b_{0, \lambda}^{*}}(0)$ is above $W^{0}(0)$. So we prefer to use a dichotomous scheme based on the standard shooting method. Let us consider the initial boundary value problem

$\forall m \leq b_{a} \quad \max _{\psi \in(0,1]}(L(\psi)-\lambda) V^{a}(m)=-\lambda V(1, m)$ and $V^{a}(0)=a, V_{m}^{a}(0)=\frac{1}{\alpha},\left(V^{a}\right)_{m}\left(b_{a}\right)=1$.

The function that associates $\left(V^{a}\right)_{m m}\left(b_{a}\right)$ to $a$ is numerically increasing for $a$ near $W^{0}(0)$ and the usual methods for finding roots may be employed here, such as Newton method. 


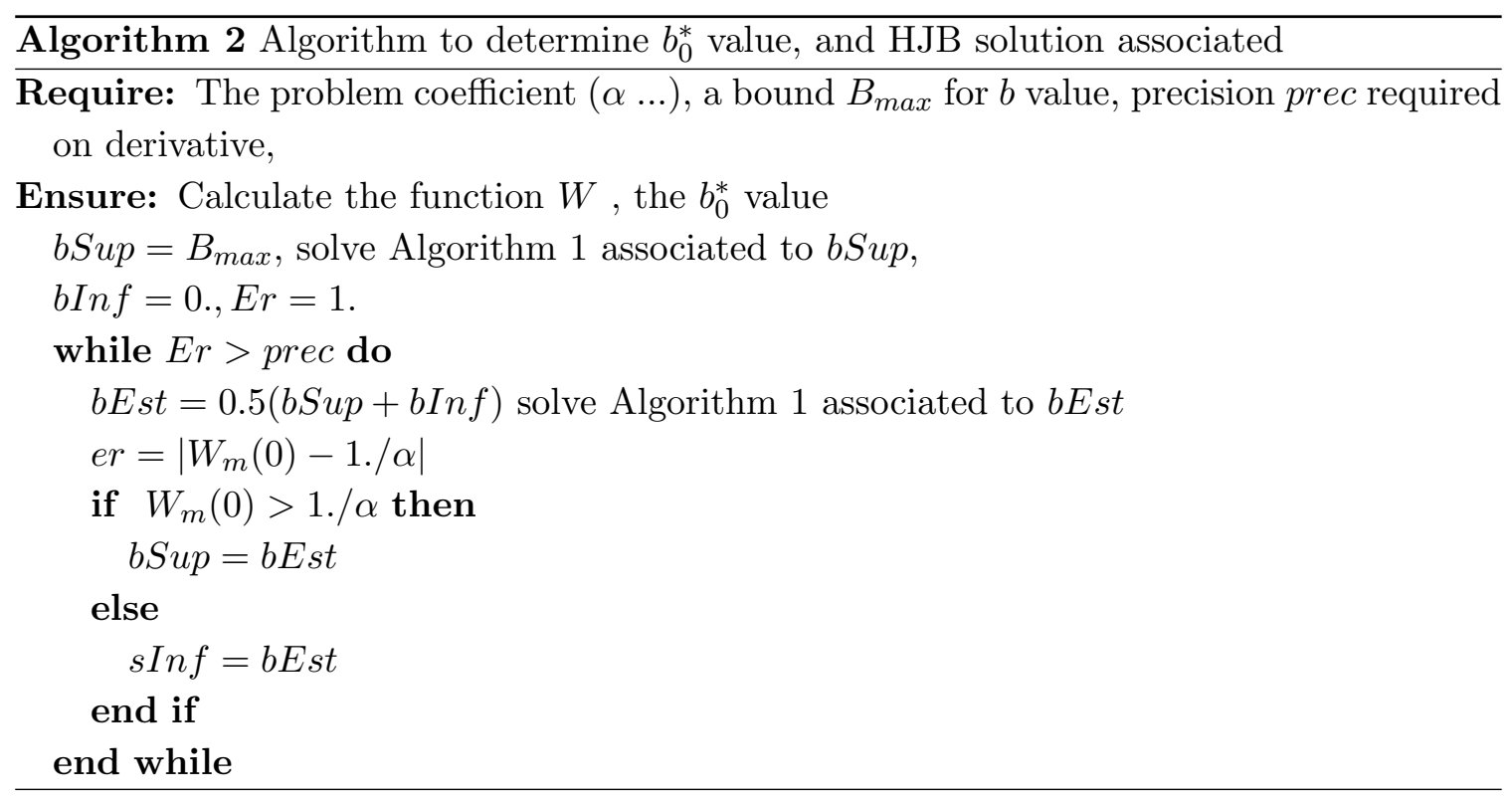

The optimal $a^{*} \operatorname{such}\left(V^{a^{*}}\right)_{m m}\left(b_{a^{*}}\right)=0$ is close to $W^{0}(0)$.

\section{$5.2 \quad$ Numerical results}

We present some results obtained for some set of parameters with the goal to exhibit some special features of the value function and the hedging strategies. Keeping the same notations as in previous sections we take the following common values for our numerical results :

- the annual continuous risk free rate $r=0.05$,

- the free cash-flow agency cost per year $\delta=0.01$,

- the annual volatility of unhedgeable risks associated to production gain $\sigma=0.2$,

- the annual volatility of hedgeable risks associated to production gain $\sigma_{h}=0.2$,

- the profitability of the cash reserves per year $\mu=0.07$,

- the equity issuance cost $\alpha=0.3$ (equivalent to a $16.66 \%$ interest rate for borrowing money),

- the investment external financing cost $\beta=0.8$ (equivalent to a $6.25 \%$ interest rate for borrowing money)

The investment cost $I$ will be a parameter taking values between 0.5 and $2, \lambda$ the opportunity occurrence intensity will evolve between 0.175 to 0.7 (meaning that the opportunity occurrence in the first year is taken equal to $16.05 \%$ and $50.34 \%$ ), and $p$ the hedging cost will vary from 0 to 0.015 euros per year for a single unit of capital stock $K_{t}$.

The effect of hedging is illustrated on figure 2 with the comparison of the value functions 
with costless hedging and without hedging. In that case, it is always optimal to invest because the function if you invest after $T$ is always above the function if you don't invest after $T$. We notice that the function values with hedging are always above the function values without hedging which means that optimal hedging policies are worthwhile. As previously shown, the value function before $T$ is always above the value function after $T$ when the decision maker decides not to invest. At a first glance, the fact that the value function before $T$ may be above the function after $T$ may seem odd : it only indicates that the opportunity is a good news for the firm and it hopes that the investment opportunity will occurs at the time where the cash level makes it very profitable.

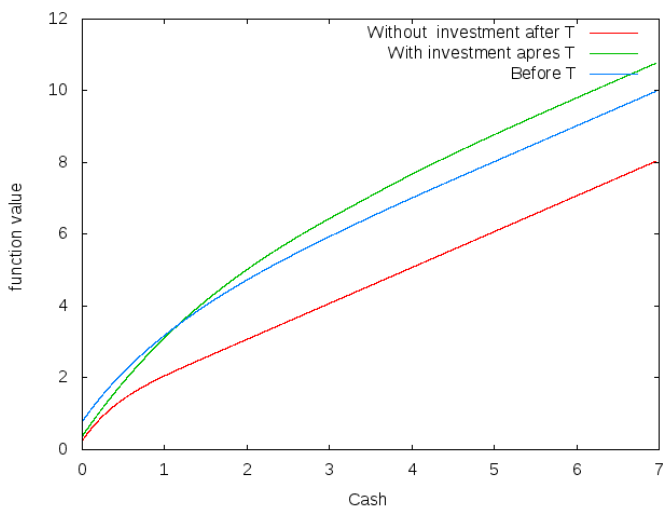

(a) No hedge

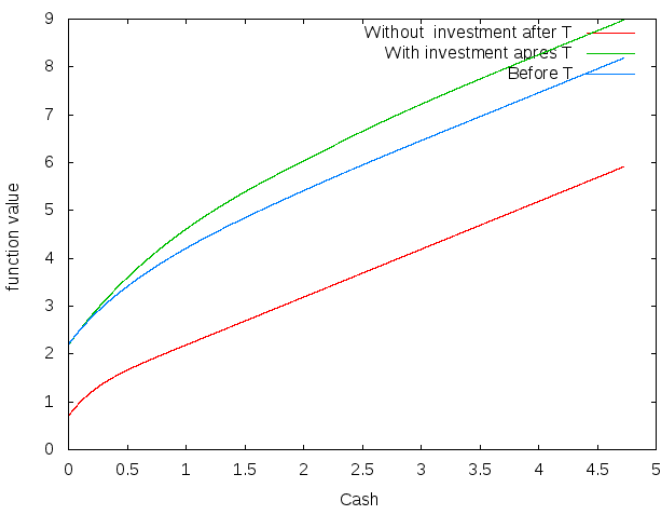

(b) Costless hedging

Figure 2: Comparing function values with and without hedge, $p=0, I=0.5, \lambda=0.175$.

When the investment cost $I$ increases, we check the obvious fact that the investment function $W^{1}$ decreases as shown on figure 2 and 3 . When the intensity of the investment

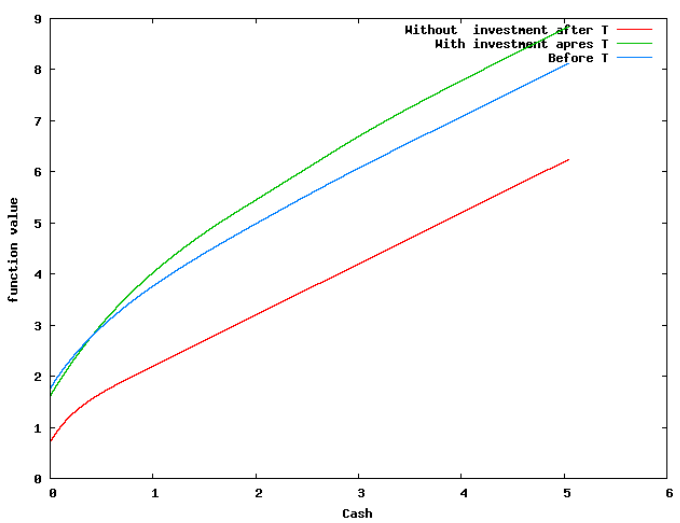

(a) $I=1$

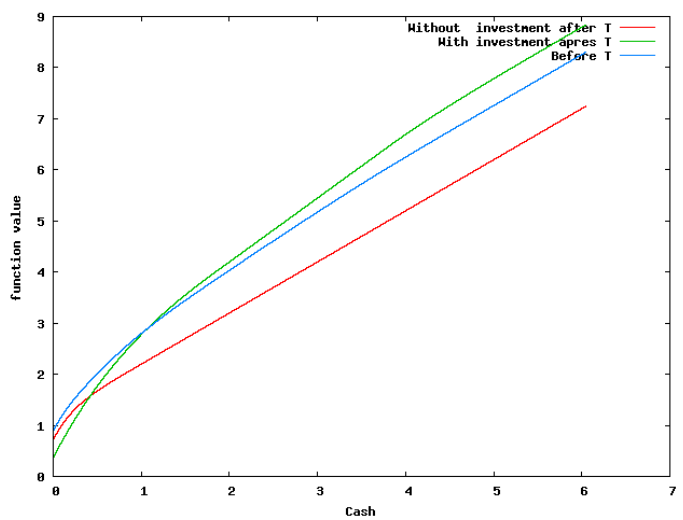

(b) $I=2$

Figure 3: Comparing function values with hedge $p=0, \lambda=0.175$ for different $I$ values

date $\lambda$ increases, the value function converges to $\max \left(W^{0}, W^{1}\right)$ as shown on figure 4 : the stochasticity of the opportunity occurrence's date vanishes (this dates goes to 0 with probability one). In the limit case where $\lambda$ is infinite, the investment opportunity is immediately available and the level of cash reserves determines the optimal decision to growth. In par- 
ticular, poor cash-firms do not invest when the two value functions $W^{0}$ and $W^{1}$ intersect.

Figure 5 gives the hedging strategy in the case of costless hedging for different values

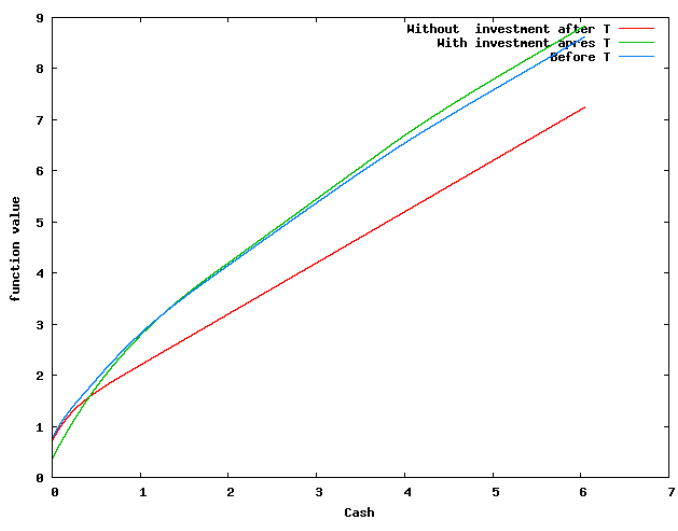

(a) $\lambda=0.525$

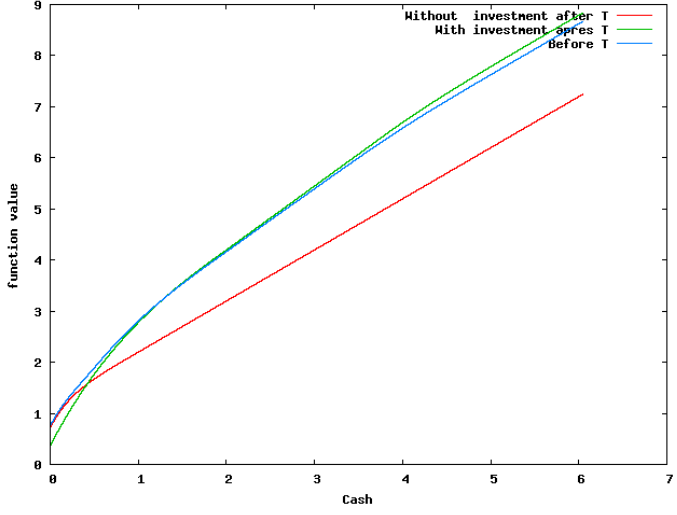

(b) $\lambda=0.7$

Figure 4: Comparing function values with hedge $p=0, I=2$ for different $\lambda$ values.

of $\lambda$. For high values of $\lambda$ the optimal hedging strategy exhibits interesting features. In particular, it is optimal to not hedge in a neighborhood of the level of cash $\tilde{m}$ for which $W^{0}(\tilde{m})=W^{1}(\tilde{m})$. This means that the value function is locally convex around $\tilde{m}$ which may be interpreted as a gamble to push the level of cash reserve to the right in order to make the investment valuable. This property highlights the nonlinear behavior of the optimal hedging strategy when there is a high chance to catch a growth opportunity. It departs from the usual precautionary role of cash reserves by adding a gambling effect due to the presence of the growth opportunity. Figure 6 shows that the firm value decreases with $p$ as

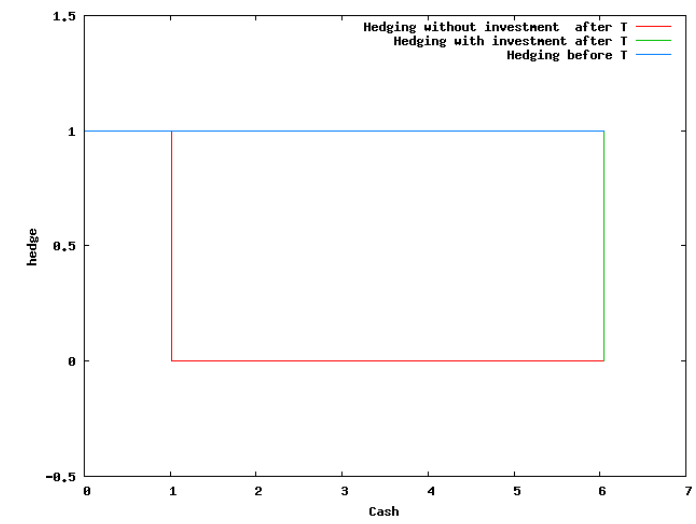

(a) $\lambda=0.175$

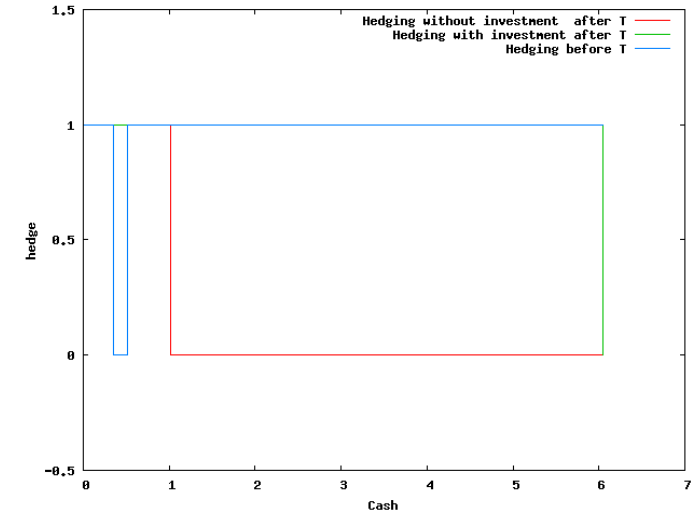

(b) $\lambda=0.7$

Figure 5: Comparing hedging strategies with $p=0, I=2$ for different $\lambda$ values.

proved in Proposition 3.2. Moreover, the non linear behavior of the hedging strategy is all the more observable that the hedging cost $p$ is high. Figure 7,8 give the optimal hedging strategies for different values of $p$. We observe that small values of $p$ have a dramatic impact on the hedging policy with the appearance of two different areas where it is optimal to not hedge while it is optimal to hedge when $p=0$. It has been checked numerically that in this 


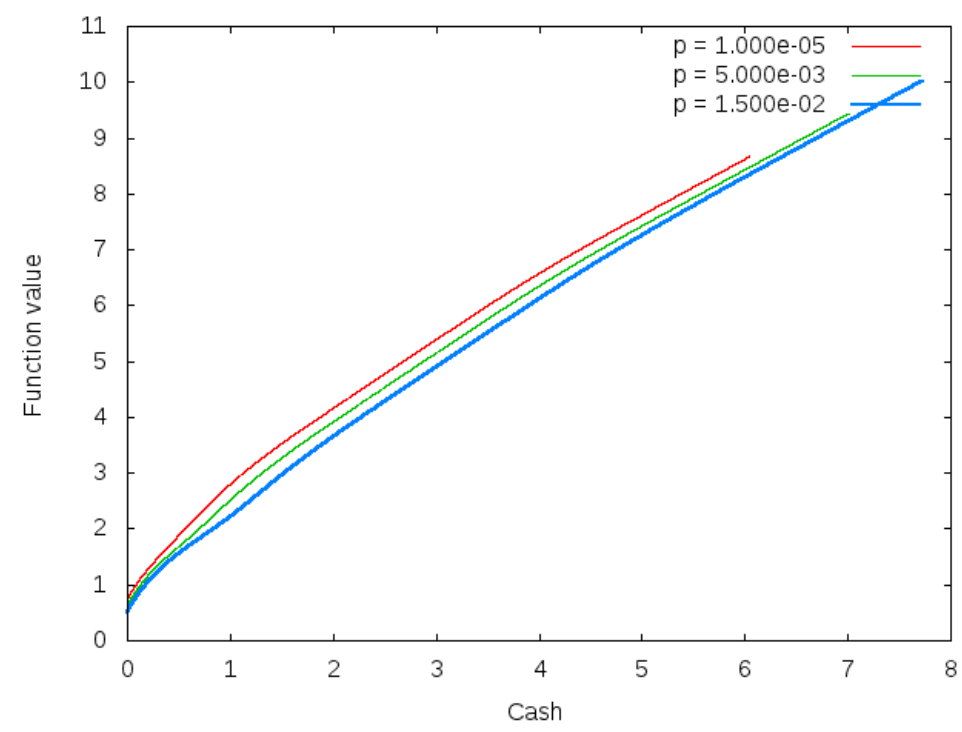

Figure 6: Comparing function values before $T$ with hedge $I=2, \lambda=0.7$.

two areas the value function is locally convex meaning that the option holder was eager for risk : the cost of hedging is more important than the expected profit of undertaking the investment with a higher cash level.

\section{Conclusion}

In this paper, we have characterized the optimal hedging policy of a liquidity constrained firm that face uncertainty on its capacity expansion. We proved that the features on the optimal liquidity management policies in terms of hedging, dividend distribution and external funding depend crucially both of the hedging costs and of the likelihood of the capacity expansion. In particular, when the likelihood of the growth opportunity is low, the shareholder value function is concave before and after the arrival of the opportunity and the hedging ratio is a decreasing function of cash level that hits zero before the threshold of dividend distribution. The novel implication of our paper is the non-monotonic feature of the hedging policy before the arrival of the opportunity when the likelihood of it is high enough. The shareholder value at the time of the appearance of the opportunity exhibits local convexity and consequently, the hedging strategy is to hedge for low level of cash reserves, to cease to hedge for intermediate levels of cash reserves in order to make profitable the potential growth opportunity, and finally to start hedging again for higher levels of cash reserves. 


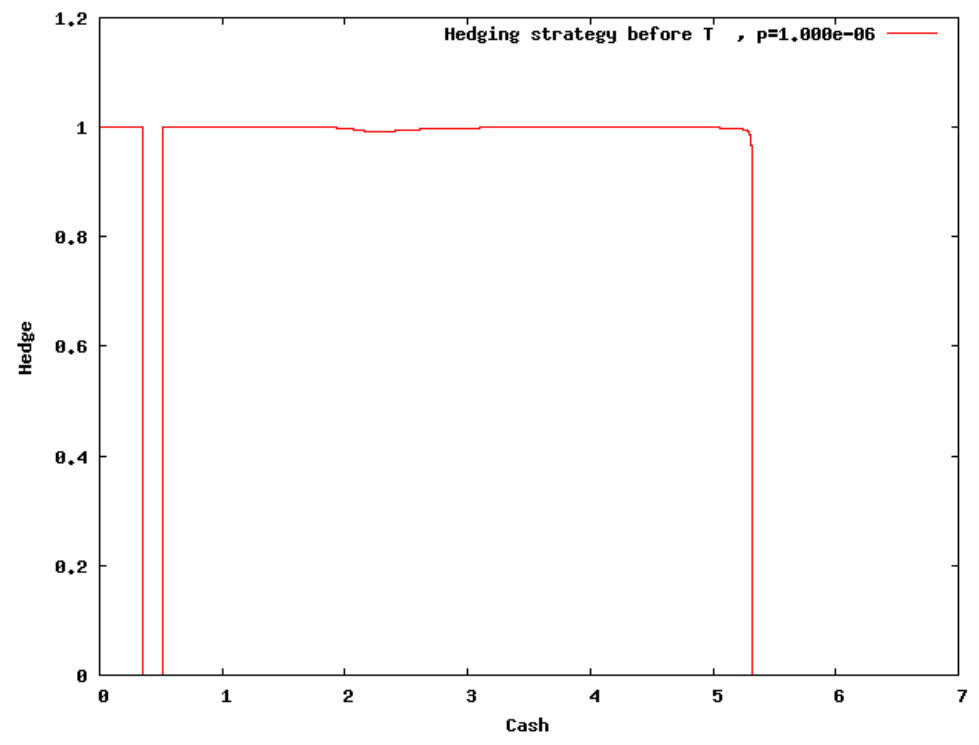

(a) $p=1 e-6$

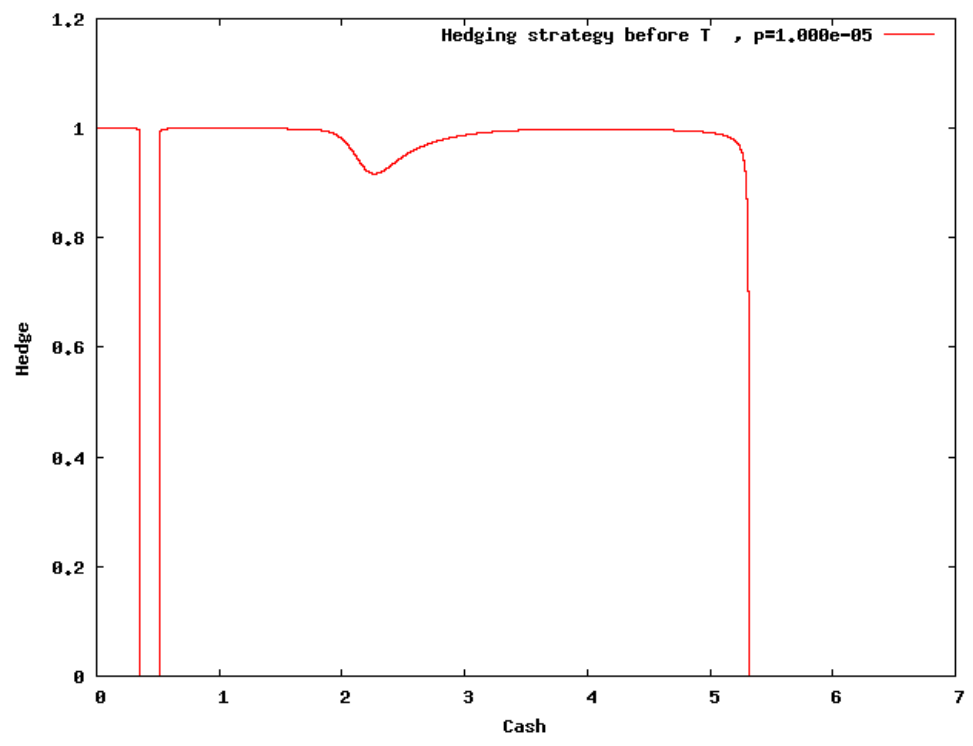

(b) $p=1 e-5$

Figure 7: Comparing hedging strategies before $T$ with $I=2 \lambda=0.7$ for $p=1 e-5$ and $p=1 e-6$. 


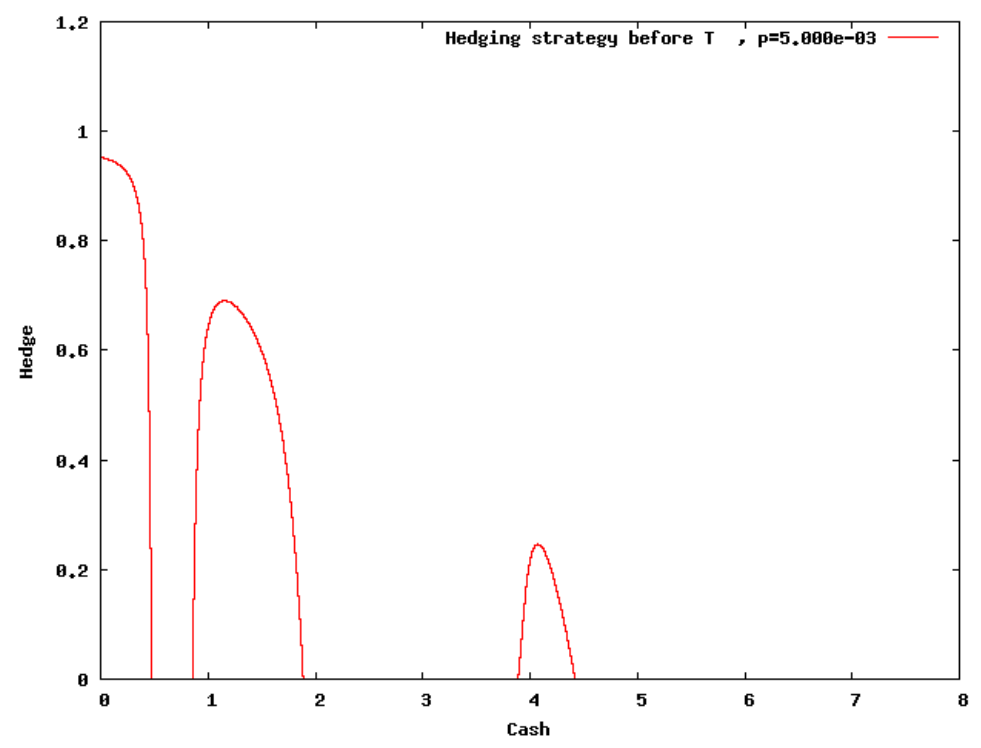

(a) $p=5 e-3$

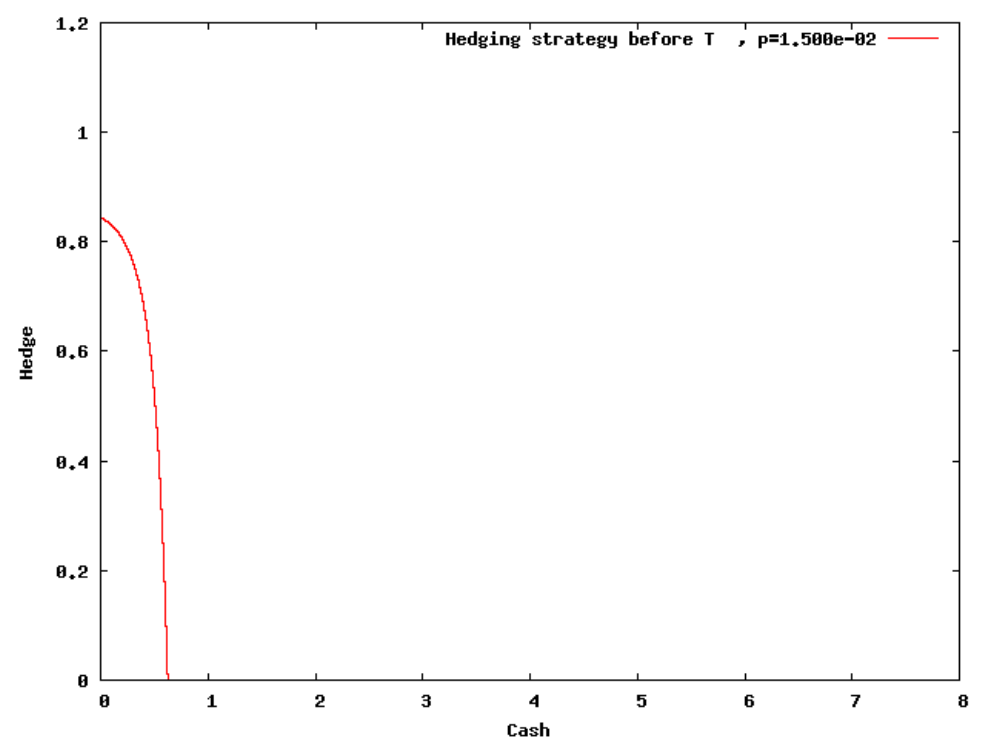

(b) $p=1 e-2$

Figure 8: Comparing hedging strategies before $T$ with $I=2 \lambda=0.7$ for $p=5 e-3$ and $p=1 e-2$. 


\section{References}

[1] Arnold, V.(1996): Equations différentielles ordinaires, Librairie du Globe.

[2] Bates, T. W., Kahle K.M. and Stulz R. (2009): Why do U.S. firms hold so much more cash than they used to? Journal of Finance 64, 1985-2021.

[3] Berk, J. and De Marzo P. (2007): Corporate Finance (Addison-Wesley, Boston, MA).

[4] Bolton, P., Chen H. and Wang N.(2011) A unified theory of Tobin's q, Corporate Investment, Financing and Risk Management. The journal of Finance, 66, 1545-1578.

[5] Boyle, G.W. and Guthrie, G.A.(2003): Investment, Uncertainty, and Liquidity. The Journal of Finance, 58, 5, 2143-2166

[6] Décamps, J.P., Mariotti, T., Rochet, J.C. and Villeneuve, S.(2011): Free cash-flows, Issuance Costs, and Stock Prices, Journal of Finance.

[7] Décamps, J.P. and Villeneuve, S.(2007): Optimal Dividend Policy and Growth option, Finance and Stochastics, Vol 11, p. 3-27.

[8] Dixit, A.K. and Pindyck, R.S.(1994): Investment Under Uncertainty. Princeton Univ. Press.

[9] Fleming, W.H. and Soner, M.(1993): Controlled markov processes and viscosity solutions, Springer-Verlag

[10] Guo, X. and Pham, H.(2005): Optimal partially reversible investment with entry decision and general production function Stochastic processes and their applications, Vol 115, 705-736.

[11] Højgaard, B. and Taksar, M. (1999): Controlling Risk exposure and dividend payout schemes: Insurance company example, Mathematical Finance, Vol 9, 2, 153-182.

[12] Hugonnier, J., Malamud, S. and Morellec, E. (2011): Capital supply uncertainty, cash holdings and Investment, Swiss Finance Institute Research Paper No. 11-44.

[13] Karatzas, I. and Shreve, S., (1991): Brownian Motion and Stochastic Calculus, Second Edition, Springer-Verlag

[14] Modigliani, Franco, and Merton H. Miller (1958): The cost of capital, corporation finance and the theory of investment, American Economic Review 48, 261-297.

[15] Sethi, S. and Taksar, M.I.: Optimal financing of a corporation subject to random returns, Mathematical Finance, Vol 12(2), p. 155-172. 